\title{
SUR LES RÉALISATIONS DES CORRESPONDANCES DE LANGLANDS DANS LA COHOMOLOGIE DES TOURS DE DRINFELD ET DE LUBIN-TATE
}

\author{
par \\ Thomas Hausberger
}

\begin{abstract}
Résumé. - Le cadre de cet article est celui du programme de Langlands : on réalise les correspondances de Langlands (du moins pour les supercuspidales) et de Jacquet-Langlands locales dans la cohomologie de certains espaces de modules de groupes $p$-divisibles, à savoir la tour dite de Lubin-Tate et la tour des revêtements de Drinfeld. Après avoir présenté les différents objets, de nature locale et globale (variétés de Shimura et variétés modulaires de Drinfeld), on explicite les résultats de Boyer-Harris-Taylor et de HausbergerHarris sur la cohomologie des deux tours et on indique les grandes lignes des preuves. Cela répond à une conjecture de Deligne, Drinfeld et Carayol datant de 1990. L'isomorphisme de Faltings-Fargues entre les deux tours est également évoqué ainsi que les travaux de Dat qui donne une réalisation cohomologique de la correspondance de Langlands pour les représentations dites elliptiques en travaillant dans une catégorie dérivée appropriée.
\end{abstract}

\begin{abstract}
On the realizations of the Langlands' correspondences in the cohomology of the Drinfeld and Lubin-Tate towers)

The framework of this article is the Langlands' program : the local Langlands' correspondence (for supercuspidals) and the local Jacquet-Langlands' correspondence are realized in the cohomology of some moduli spaces of $p$ divisible groups, precisely the so-called Lubin-Tate tower and the tower of Drinfeld's coverings. After presenting the different objects, of local and global (the Shimura varieties and Drinfeld's modular varieties) nature, results by Boyer-Harris-Taylor and Harris-Hausberger on the cohomology of the two towers are described and the main lines of the proofs are sketched. This responds to a conjecture of Deligne, Drinfeld and Carayol dating from 1990. The Faltings-Fargues isomorphism between the two towers is also mentionned as well as the work of Dat who gives a cohomological realization of the Langlands' correspondence for the so-called elliptic representations, working in an appropriate derived category.
\end{abstract}

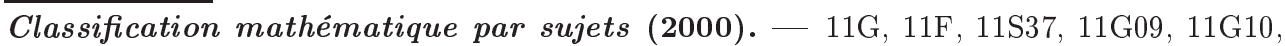
11G18, 11G35, 11R39, 14G22, 14G35, 14L05. 
Table des matières

Introduction................................... 2

1. Le programme de Langlands..................... 3

2. Les deux tours.............................. 5

3. L'isomorphisme de Faltings-Fargues................ 11

4. Les acteurs globaux......................... 13

5. Les suites spectrales........................... 19

6. Réalisations géométriques des correspondences........... 21

7. Calcul de la cohomologie non supercuspidale.......... 27

8. Réalisation cohomologique de la correspondance de Langlands pour les représentations elliptiques......... 35

9. Perspectives.................................. 38

Références................................... 39

\section{Introduction}

Cet article d'exposition fait suite à celui de Henri Carayol : on y expose les résultats récents mentionnés en fin de texte. Il s'agit donc toujours de la réalisation du "programme de Carayol" décrit dans [17] (sur une idée de Deligne [26]). L'accent est mis sur les deux représentations "locales fondamentales" qui réalisent les correspondances de Langlands et de Jacquet-Langlands locales, donc sur les tours de Lubin-Tate et de Drinfeld (et les liens qui les unissent), dont on considère la cohomologie étale $\ell$-adique à support compact. L'aboutissement est le théorème 8 qui décrit totalement cette cohomologie. Les détails seront donnés dans le cas des corps de fonctions (celui des corps de nombres est, dans les grandes lignes, similaire), à la fois parce que l'auteur est davantage familier des variétés modulaires de Drinfeld et du fait que les variétés de Shimura ont déjà trouvé bonne place dans l'article de Carayol.

Des rappels historiques sont fournis et les contributions des différents auteurs précisés au cours du texte. Figurent aussi, autant que faire se peut, les grandes lignes des preuves. Face à la grande quantité de littérature que cela représente, l'auteur de ce texte sollicite l'indulgence du lecteur quant aux raccourcis et simplifications auxquelles il a dû opérer pour rendre ce texte concis et lisible, raccourcis qui ont pour résultats d'inévitables imprécisions. 


\section{Le programme de Langlands}

Nous allons faire quelques rappels concernant la correspondance de Langlands locale pour le groupe linéaire, ainsi que la correspondance de JacquetLanglands locale qui est une instance des diverses fonctorialités prévues par Langlands, les deux correspondances apparaissant de paire dans la cohomologie des variétés globales que nous présenterons plus loin dans cet article.

On se donne donc $K$ un corps local non archimédien, c'est-à-dire soit une extension finie $K / \mathbb{Q}_{p}$ de corps résiduel $k=\mathbb{F}_{q}$, soit un corps de séries formelles de Laurent en une indéterminée $K=\mathbb{F}_{q}((t))$. Comme de coutume, on note $\mathcal{O}$ son anneau des entiers et $\varpi$ une uniformisante.

1.1. La correspondance de Jacquet-Langlands locale. - Soit $D_{d}$ "l"' algèbre à division de centre $K$ et d'invariant $1 / d$. Désignons par $\mathcal{A}^{\prime}(d)$ l'ensemble des (classes d'équivalence de) représentations complexes admissibles irréductibles du groupe $D_{d}^{\times}$(ces représentations sont de dimension finie). D'autre part, soit $\mathcal{A}(d)$ l'ensemble des (classes d'équivalence de) représentations complexes admissibles irréductibles du groupe $\mathrm{GL}_{d}(K)$. On note $\mathcal{A}^{2}(d)$ le sousensemble constitué des représentations qui sont essentiellement de carré intégrable (i.e. les coefficients matriciels sont intégrables modulo le centre), lequel contient l'ensemble $\mathcal{A}^{0}(d)$ des représentations cuspidales (leurs coefficients sont à support compact modulo le centre).

Tant les éléments de $\mathcal{A}(d)$ que ceux de $\mathcal{A}^{\prime}(d)$ admettent des caractères (pour les premiers, ils sont définis sur les éléments semi-simples réguliers).

Théorème 1. - Il existe une famille de bijections $(d \geq 1)$ :

$$
\mathrm{JL}_{d}: \mathcal{A}^{2}(d) \rightarrow \mathcal{A}^{\prime}(d), \pi \mapsto \mathrm{JL}(\pi)
$$

caractérisée par l'égalité (au signe près) des caractères $\chi_{\pi}(g)=(-1)^{d-1} \chi_{\mathrm{JL}(\pi)}\left(g^{\prime}\right)$ sur les éléments réguliers associés (i.e. qui ont même polynôme minimal).

Ce théorème a été démontré, dans le cas des corps $p$-adiques, par Jacquet et Langlands (pour $d=2$; [52]), puis par Rogawski, Kazhdan et Vignéras (pour $d$ quelconque; [64] [24]). Il est dû à Badulescu en égale caractéristique (voir [1] ou [2] pour une généralisation). Les preuves procédent par voie globale et reposent sur la formule des traces de Selberg.

Dans ce qui suit, on considérera non pas des représentations complexes mais à valeur dans $\overline{\mathbb{Q}}_{\ell}(\ell \neq p)$. La notion de représentation essentiellement de carré intégrable est une notion "algébrique" (c'est évident pour les cuspidales; pour les autres, cela résulte du cas cuspidal, du théorème de classification de Zelevinsky 
des séries discrètes pour $\mathrm{GL}_{d}$ et de considérations sur la rationalité du processus d'induction). On peut donc transporter la bijection du théorème précédent, via l'isomorphisme $\overline{\mathbb{Q}}_{\ell} \simeq \mathbb{C}$ choisi : la correspondance de Jacquet-Langlands locale est équivariante sous l'action de $\operatorname{Aut}(\mathbb{C})$ parce que les caractères des représentations le sont. Désormais, les éléments de $\mathcal{A}(d)$ et $\mathcal{A}^{\prime}(d)$ seront vues comme des $\overline{\mathbb{Q}}_{\ell}$-représentations.

1.2. La correspondance de Langlands locale. - On considère maintenant le groupe de Weil $W_{K}$, constitué des éléments du groupe de Galois absolu $\operatorname{Gal}(\bar{K} / K)$ qui induisent une puissance entière du Frobenius $\operatorname{Fr}_{q}$ sur le corps résiduel $\bar{k}$ de l'extension maximale non ramifiée $K^{n r}$ ( $\bar{K}$ désigne bien entendu une clôture séparable en égale caractéristique). Ce dernier s'insère donc dans une suite exacte

$$
1 \rightarrow I_{K} \rightarrow W_{K} \rightarrow<\operatorname{Fr}_{q}>\simeq \mathbb{Z} \rightarrow 1,
$$

où $I_{K}=\operatorname{Gal}\left(\bar{K} / K^{n r}\right)$ désigne le sous-groupe d'inertie, et il est doté de la topologie pour laquelle $I_{K}$, muni de sa topologie de Krull, est un sous-groupe ouvert et le quotient $W_{K} / I_{K}$ est discret.

Pour tout entier $d \geq 1$, nous désignons par $\mathcal{G}(d)$ l'ensemble des (classes d'équivalences de) représentations complexes de Weil-Deligne continues de degré $d$ Frob-semi-simples du groupe $W_{K}$. Précisément, il s'agit d'un couple $(\rho, N)$, où $\rho$ est une représentation complexe continue semi-simple de dimension $d$ de $W_{K}$ (son espace $E$ est doté de la topologie discrète) et $N$ un endomorphisme nilpotent de $E$ qui commute avec l'action de l'inertie et tel que $\rho(F r) N=$ $q^{-1} N \rho(F r)$. On a noté $F r$ un élément de Frobenius "géomètrique", qui induit $\mathrm{Fr}_{q}^{-1}$ sur le corps résiduel $\bar{k}$. Nous considérons également le sous-ensemble $\mathcal{G}^{2}(d)$ des représentations indécomposables, qui contient celui $\mathcal{G}^{0}(d)$ des représentations irréductibles (cela implique $N=0$ ).

Théorème 2. - Il existe une famille de bijections $(d \geq 1, i \in\{\emptyset, 2,0\})$ :

$$
\mathrm{L}_{d}: \mathcal{A}_{\ell}^{i}(d) \rightarrow \mathcal{G}_{\ell}^{i}(d), \pi \mapsto \mathrm{L}(\pi)
$$

caractérisée par la compatibilité à la théorie du corps de classe (avec laquelle elle coïncide pour $d=1$, la normalisation étant telle que l'uniformisante $\varpi$ corresponde au Frobenius géométrique Fr), la compatibilité à la torsion par un quasi-caractère et au passage à la contragrédiente, et la préservation des fonctions $L$ et facteurs $\epsilon$ de paires.

Ce théorème célèbre a été démontré par Kutzo pour $d=2$ ([53]) puis par Henniart pour $d=3$ ([48]). Pour $d$ quelconque, il a été d'abord démontré par 
Laumon, Rapoport et Stuhler en égale caractéristique ([54]) puis, dans le cas p-adique, indépendemment par Henniart d'une part ([49]; voir également [51] pour l'unicité) et Harris et Taylor ([46]) d'autre part, après un résultat partiel de Harris ([42]). Le lecteur pourra consulter également les exposés de Carayol [19] et [20] au séminaire Bourbaki.

Remarque 1. - Bien que la correspondance de Langlands locale soit établie dès qu'elle l'est pour les cuspidales, qui constituent les briques élémentaires au vu de la classification de Bernstein et Zelevinsky ([9], [69] ; voir également l'article d'exposition [63]), la preuve de Harris et Taylor passe par une construction de la correspondance pour $\pi$ générale (c'est nécessaire afin de démontrer la préservation des facteurs $\epsilon$ de paires).

A vrai dire, le théorème précédent est démontré non pas pour des représentations à valeurs complexes, mais pour des représentations $\ell$-adiques, et c'est pour cela que le formalisme de Weil-Deligne a été introduit ([25]). La géométrie des variétés modulaires globales dont il sera question plus tard fournit $\left(\right.$ sur $\left.\overline{\mathbb{Q}}_{\ell}\right)$ la correspondance dite "de Hecke" $r_{d}(\pi)=\mathrm{L}_{d}\left(\pi^{\vee}\right) \otimes||^{\frac{1-d}{2}}$, qui est invariante par $\operatorname{Aut}(\mathbb{C})$ (voir [50]). On peut donc transporter la bijection via l'isomorphisme $\overline{\mathbb{Q}}_{\ell} \simeq \mathbb{C}$ choisi.

A partir de maintenant, on regardera $\mathcal{A}_{d}^{i}(K)$ et $\mathcal{G}_{d}^{i}(K)$ comme des ensembles de $\overline{\mathbb{Q}}_{\ell}$-représentations. On peut alors oublier $N$ dans la définition (on considère donc les représentations $\ell$-adiques continues de dimension $d$ de $W_{K}$ telles que l'action du Frobenius soit semi-simple), car on récupère cet opérateur appelé monodromie par le théorème de monodromie $\ell$-adique de Grothendieck.

Lorsque l'on s'intéresse aux réalisations géométrico-cohomologiques de ces correspondances, les objets locaux à considérer sont les tours de Lubin-Tate et de Drinfeld.

\section{Les deux tours}

On poursuit avec les notations précédentes et désigne par $\widehat{K}^{n r}$ le complété de l'extension maximale non-ramifiée de $K$ et $\widehat{\mathcal{O}}^{n r}$ son anneau des entiers. On note également $\mathcal{O}_{D_{d}}$ l'ordre maximal du corps gauche $D_{d}$ et $\mathcal{O}_{d} \subset \mathcal{O}_{D_{d}}$ l'anneau des entiers de l'extension non ramifiée de degré $d$. Ainsi $\mathcal{O}_{D_{d}}$ s'identifie-t-il à l'algèbre $\mathcal{O}_{d}[\Pi]$ soumises aux relations $\Pi^{d}=\varpi$ et $\Pi a=a^{q} \Pi\left(a \in \mathcal{O}_{d}\right)$.

On rappelle qu'un $\mathcal{O}$-module formel $X$ (défini sur une $\mathcal{O}$-algèbre $B$ dans laquelle l'image de $\pi$ est nilpotente) est un groupe formel muni d'une action 
de $\mathcal{O}$ induisant l'action naturelle sur son algèbre de Lie. Un $\mathcal{O}_{D_{d}}$-module formel est un $\mathcal{O}$-module formel muni d'une action de $\mathcal{O}_{D_{d}}$ prolongeant celle de $\mathcal{O}$. Il est dit spécial si l'action de $\mathcal{O}_{d}$ sur $\operatorname{Lie}(X)$ est telle qu'elle décompose $\operatorname{Lie}(X)=\oplus_{i \in \mathbb{Z} / d \mathbb{Z}} \operatorname{Lie}(X)_{i}$ selon les $d$ plongements $\mathcal{O}_{d} \hookrightarrow \bar{K}$ en $d$ composantes toutes de rang 1. En d'autres termes, on demande que $\operatorname{Lie}(X)$ soit un $\mathcal{O}_{d} \otimes B$-module localement libre de rang 1 (définition due à Drinfeld). Enfin, une isogénie de $\mathcal{O}$-modules formels est une isogénie de groupes formels $\mathcal{O}$-équivariante. Sa hauteur est la $\mathcal{O}$-hauteur de l'isogénie $X(\varpi)$, c'est-à-dire l'entier $h$ tel que $X(\varpi)=t^{q^{h}}+a_{h-1} t^{q^{h-1}}+\ldots+a_{1} t^{q}+\varpi t$. Une quasi-isogénie $X_{1} \rightarrow X_{2}$ est un élément dans $\operatorname{Hom}\left(X_{1}, X_{2}\right) \otimes_{\mathcal{O}} K$ qui possède un inverse (dans $\left.\operatorname{Hom}\left(X_{1}, X_{2}\right) \otimes_{\mathcal{O}} K\right)$. C'est une isogénie à multiplication près par une puissance de $X_{2}(\varpi)$, ce qui permet d'en définir la hauteur. Un $\mathcal{O}_{D_{d}}$-module formel spécial est de dimension $d$ et hauteur $d^{2}$.

Nous allons, pour chaque objet, en donner la présentation "classique" ([19]) puis la description selon le formalisme de Rapoport-Zink ([60]), qui en constitue le cadre naturel, pour la comparaison ([39]) et la généralisation.

2.1. La tour des revêtements de Drinfeld. - Tout commence avec le demi-plan $\varpi$-adique généralisé de Drinfeld ([28]) :

$$
\Omega^{d}=\mathbb{P}^{d-1}-\bigcup_{H / K} H
$$

où $H$ parcourt l'ensemble des hyperplans de $\mathbb{P}^{d-1}$ rationnels sur $K$. C'est un analogue non-archimédien des espaces hermitiens symétriques, pour le cas du groupe linéaire $\mathrm{GL}_{d}$. Pour $d=2$, on retrouve l'analogue, déjà considéré par Tate $([67])$, du double demi-plan de Poincaré $\mathcal{H}^{ \pm}=\mathbb{P}^{1}(\mathbb{C})-\mathbb{P}^{1}(\mathbb{R})$.

La variété $\Omega^{d}$ est munie d'une structure d'espace rigide-analytique. Elle possède un modèle formel $\widehat{\Omega}^{d}$, construit par Deligne, dont $\Omega^{d}$ est donc la fibre générique au sens de Raynaud ([62]) et sur lequel agit $\operatorname{PGL}_{d}(K)$. Fixons $\Phi$ un $\mathcal{O}_{D_{d}}$ module formel spécial sur $\bar{k}$ (ils sont tous isogènes). Drinfeld a découvert que $\widehat{\Omega}^{d} \hat{\otimes} \widehat{\mathcal{O}}^{n r}$ classifie, sur les $\widehat{\mathcal{O}}^{n r}$-algèbre s où l'image de $\varpi$ est nilpotente, les

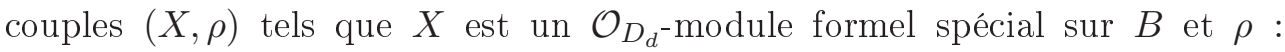
$\Phi_{\bar{B}} \rightarrow X_{\bar{B}}$ est une quasi-isogénie de hauteur zéro (on note $\bar{B}=B / \varpi^{n} B$ et rigidifie donc la fibre spéciale uniquement). Soit maintenant $\mathbb{X}$ le $\mathcal{O}_{D_{d}}$-module formel universel au-dessus de $\widehat{\Omega}^{d} \hat{\otimes} \widehat{\mathcal{O}}^{n r}$. La fibre générique $\chi_{n}^{d}$ du noyau de la multiplication par $\varpi^{n}$ est localement pour la topologie étale isomorphe à $\Omega^{d} \times \mathcal{O}_{D_{d}} / \varpi^{n} \mathcal{O}_{D_{d}}$. On définit alors, suivant Drinfeld ([29]), les revêtements

$$
\Sigma_{n}^{d}=\operatorname{Isom}_{\mathcal{O}_{D_{d}}}\left(\varpi^{-n} \mathcal{O}_{D_{d}} / \mathcal{O}_{D_{d}}, \chi_{n}^{d}\right)
$$


de $\Omega^{d} \hat{\otimes} \widehat{K}^{n r}$, qui sont des revêtements étales galoisiens de groupe $\left(\mathcal{O}_{D_{d}} / \varpi^{n} \mathcal{O}_{D_{d}}\right)^{\times}$. Le groupe $\mathrm{GL}_{d}(K)$, identifié à $\operatorname{Aut}_{\mathcal{O}_{D_{d}}}(\Phi) \otimes_{\mathcal{O}} K$, agit via l'isogénie $\rho$ et se relève aux revêtements. Cela correspond à l'action naturelle sur $\widehat{\Omega}^{d}$ tordue par $g \mapsto F r_{q}^{-v(\operatorname{det} g)}$ sur $\widehat{K}^{n r}$. Enfin, les $\Sigma_{n}^{d}$ constituent un système projectif via les inclusions $\varpi^{-m} \mathcal{O}_{D_{d}} / \mathcal{O}_{D_{d}} \subset \varpi^{-n} \mathcal{O}_{D_{d}} / \mathcal{O}_{D_{d}}$ pour $m \leq n$. Le groupe $D_{d}^{\times}$agit sur la base de la tour par $d \mapsto F r_{q}^{-v(\operatorname{Nrd} d)}$, où Nrd : $D^{\times} \rightarrow K^{\times}$ désigne la norme réduite, et se relève aux revêtements de façon compatible à l'action de $\mathcal{O}_{D_{d}}^{\times}$sur la tour. En définitive, le groupe $D_{d}^{\times}$agit sur la tour par "correspondances" et cette action commute avec celle de $\mathrm{GL}_{d}(K)$ sur chaque étage.

Il est commode pour ce qui suit de prendre des hauteurs de hauteur abitraire, dans l'esprit du formalisme de Rapoport-Zink, ce qui permet également de simplifier la description des actions. Nous transformons au passage l'action à gauche de Drinfeld en une action à droite (afin d'obtenir au final une action à gauche sur la cohomologie $\ell$-adique). On obtient un $\widehat{\mathcal{O}}^{n r}$-schéma formel $\widehat{\mathcal{M}}_{\mathcal{D} r, 0}^{d}$, muni d'une action à droite de $\mathrm{GL}_{d}(K)$, qui se décompose en $\widehat{\mathcal{M}}_{\mathcal{D} r, 0}^{d}=\bigsqcup_{h \in \mathbb{Z}} \widehat{\mathcal{M}}_{\mathcal{D} r, 0}^{d,[h]}$, où $\widehat{\mathcal{M}}_{\mathcal{D} r, 0}^{d,[h]}$ désigne la composante connexe où la quasiisogénie $\rho$ est de hauteur $d h$. On a donc $\widehat{\mathcal{M}}_{\mathcal{D} r, 0}^{d,[0]}=\widehat{\Omega}^{d} \hat{\otimes} \widehat{\mathcal{O}}^{n r}$ (mais les actions ne se comparent pas directement). Le schéma $\widehat{\mathcal{M}}_{\mathcal{D} r, 0}^{d}$ est muni d'une donnée de descente non effective à $\operatorname{Spf}(\mathcal{O}): \phi: \widehat{\mathcal{M}}_{\mathcal{D} r, 0}^{d} \stackrel{\sim}{\rightarrow}\left(\widehat{\mathcal{M}}_{\mathcal{D} r, 0}^{d}\right)^{\operatorname{Fr}_{q}}$, qui envoie $\widehat{\mathcal{M}}_{\mathcal{D} r, 0}^{d,[h]}$ $\operatorname{sur}\left(\widehat{\mathcal{M}}_{\mathcal{D} r, 0}^{d,[h-1]}\right)^{\operatorname{Fr}_{q}}$. Soit $\mathcal{M}_{\mathcal{D} r, 0}^{d}$ la fibre générique de $\widehat{\mathcal{M}}_{\mathcal{D} r, 0}^{d}$; à l'aide de structures de niveau $\eta$, on définit une tour de revêtements étales $\mathcal{M}_{\mathcal{D} r, U}^{d}$ indexée par les sous-groupes ouvert $U$ de $\mathcal{O}_{D_{d}}^{\times}$, munie d'une action à droite de $\mathrm{GL}_{d}(K) \times D_{d}^{\times}$ définie par

$$
(g, d): \mathcal{M}_{\mathcal{D} r, U}^{d} \rightarrow \mathcal{M}_{\mathcal{D} r, d^{-1} U d}^{d},(X, \rho, \eta) \mapsto(X, \rho \circ g, \eta \circ d) .
$$

Si l'on pose $U_{n}=1+\varpi^{n} \mathcal{O}_{D_{d}}$, on retrouve $\mathcal{M}_{\mathcal{D} r, U_{n}}^{d,[0]}=\Sigma_{n}^{d}$. L'action est telle que $(g, d)$ envoie $\mathcal{M}_{\mathcal{D} r, U}^{d,[h]} \operatorname{sur} \mathcal{M}_{\mathcal{D} r, d U d^{-1}}^{d,[h+v(\operatorname{det} g)+v(\operatorname{Nrd} d)]}$. Cela conduit à définir le noyau $\left(\mathrm{GL}_{d}(K) \times D_{d}^{\times} \times W_{K}\right)^{0}$ du caractère surjectif

$$
\begin{aligned}
\mathrm{GL}_{d}(K) \times D_{d}^{\times} \times W_{K} & \rightarrow \mathbb{Z} \\
(g, d, w) & \mapsto v(\operatorname{det} g)+v(\operatorname{Nrd} d)+v(c l(w))
\end{aligned}
$$

où $c l: W_{K} \rightarrow K^{\times}$désigne le morphisme du corps de classes. Ce sous-groupe agit sur $\mathcal{M}_{\mathcal{D} r, U}^{d,[0]}$; en terme de représentations, la cohomologie de $\mathcal{M}_{\mathcal{D} r, U_{n}}^{d}$ est l'induite de $\left(\mathrm{GL}_{d}(K) \times D_{d}^{\times} \times W_{K}\right)^{0}$ à $\mathrm{GL}_{d}(K) \times D_{d}^{\times} \times W_{K}$ de la cohomologie de $\Sigma_{n}^{d}$. L'action de $K^{\times}$plongé centralement dans $\mathrm{GL}_{d}(K) \times D_{d}^{\times}$par $x \mapsto\left(x, x^{-1}\right)$ 
est triviale. Noter également que l'action de $W_{K}$ est définie via la donnée de descente, qui n'est effective qu'en quotientant par le sous-groupe engendré par une puissance de $\varpi$, ce qui revient au niveau de la cohomologie à fixer un caractère central d'ordre fini (ce que nous ferons, voir théorème 8).

2.2. La tour de Lubin-Tate. - On s'intéresse maintenant au foncteur des déformations par quasi-isogénie d'un $\mathcal{O}$-module formel $\Phi_{d}$ de hauteur $d$ et dimension 1, cas étudié (lorsque la quasi-isogénie est de hauteur zéro, donc un isomorphisme) originellement par Lubin et Tate ([56]) puis Drinfeld ([28]; il s'en sert essentiellement pour démontrer la lissité des variétés de modules elliptiques). On obtient le $\widehat{\mathcal{O}}^{n r}$-schéma formel $\widehat{\mathcal{M}}_{\mathcal{L} \mathcal{T}, 0}^{d}$, muni d'une action de $D_{d}^{\times}$ identifié au groupe des quasi-isogénies de $\Phi_{d}$. On a une décomposition $\widehat{\mathcal{M}}_{\mathcal{L} T \text {, } 0}^{d}=$ $\bigsqcup_{h \in \mathbb{Z}} \widehat{\mathcal{M}}_{\mathcal{L} T, 0}^{d,[h]}$, où $\widehat{\mathcal{M}}_{\mathcal{L} \mathcal{T}, 0}^{d,[h]}$ désigne la composante où la quasi-isogénie $\rho$ est de hauteur $h$. On retrouve en degré 0 le résultat de Lubin-Tate-Drinfeld : $\widehat{\mathcal{M}}_{\mathcal{L} T \text {, }, 0}^{d,[0]}=$ $\operatorname{Spf}\left(\widehat{\mathcal{O}}^{n r}\left[\left[t_{1}, \ldots, t_{d-1}\right]\right]\right)$. La fibre générique $\mathcal{M}_{\mathcal{L} \mathcal{T}, 0}^{d}$ est donc isomorphe, en tant qu'espace rigide-analytique, à $\mathbb{Z}$ copies de la boule unité $\stackrel{\circ}{B}^{d-1}$.

Comme dans le cas précédent, on peut définir une tour indexée par les sousgroupes ouverts de $\mathrm{GL}_{d}(\mathcal{O})$. Mieux, à l'aide de la notion de structure de niveau de Drinfeld, on peut définir des structures entières (la structure de niveau était uniquement en fibre générique dans le cas précédent) : si $X$ est un $\mathcal{O}$-module formel sur $B$, il s'agit d'un morphisme de $\mathcal{O}$-modules $\eta:\left(\varpi^{-n} \mathcal{O} / \mathcal{O}\right)^{d} \rightarrow \mathfrak{m}_{B}$, où $\mathfrak{m}_{B}$ désigne l'idéal maximal de $B$, tel que $\prod_{x \in \varpi^{-n} \mathcal{O} / \mathcal{O}}(t-\eta(x))$ divise la série formelle donnant l'action de $\varpi \operatorname{sur} X$. On obtient des schémas formels $\widehat{\mathcal{M}}_{\mathcal{L} T, n}^{d}$.

Revenons en fibre générique : la tour $\mathcal{M}_{\mathcal{L} \mathcal{T}, U}^{d}$ est munie d'une action à droite par correspondances de $\mathrm{GL}_{d}(F)$ où pour $g \in \mathrm{GL}_{d}(F)$ tel que $g^{-1} U g \subset \mathrm{GL}_{d}(\mathcal{O})$ on a $g: \mathcal{M}_{\mathcal{L} \mathcal{T}, U}^{d} \stackrel{\sim}{\rightarrow} \mathcal{M}_{\mathcal{L} \mathcal{T}, g^{-1} U g}^{d}$, d'une action à droite de $D_{d}^{\times}$sur chaque étage (légère modification par rapport au formalisme de Rapoport-Zink qui choisissent cette action à gauche ; les deux points de vue différent par $d \mapsto d^{-1}$ ), et d'une donnée de descente à la Weil (non effective). Décomposant la tour en $\mathcal{M}_{\mathcal{L} \mathcal{T}, U}^{d}=\bigsqcup_{h \in \mathbb{Z}} \mathcal{M}_{\mathcal{L} \mathcal{T}, U}^{d,[h]}$ et définissant le noyau $\left(\mathrm{GL}_{d}(K) \times D_{d}^{\times} \times W_{K}\right)^{1} \mathrm{du}$ caractère surjectif

$$
\begin{aligned}
\mathrm{GL}_{d}(K) \times D_{d}^{\times} \times W_{K} & \rightarrow \mathbb{Z} \\
(g, d, w) & \mapsto-v(\operatorname{det} g)+v(\operatorname{Nrd} d)+v(c l(w))
\end{aligned}
$$


l'action sur $\mathcal{M}_{\mathcal{L} \mathcal{T}}^{d}$ est géométriquement induite de celle de $\left(\mathrm{GL}_{d}(K) \times D_{d}^{\times} \times\right.$ $\left.W_{K}\right)^{1}$ sur $\mathcal{M}_{\mathcal{L} \mathcal{T}}^{d,[0]}$. Le sous-groupe $K^{\times}$, plongé diagonalement dans $\mathrm{GL}_{d}(K) \times$ $D_{d}^{\times}$, agit trivialement.

2.3. Les représentations locales fondamentales. - Il existe différentes théories des espaces rigides-analytiques, depuis la définition de Tate ([67]; noter que ces espaces apparaissent d'emblée comme le contexte naturel où a lieu l'uniformisation de certaines courbes elliptiques) et la vision de Raynaud en tant que fibre générique de schémas formels ([62]). Nous choisissons la théorie de Berkovich, qui est une des plus riches de part le formalisme cohomologique étale élaboré ([4]), Berkovich prouvant également les théorèmes de comparaison de type GAGA (géométrie algébrique - géométrie analytique; [6]), de changement de base, ..., que l'on peut souhaiter. Une des motivations de Berkovich a d'ailleurs été, suite aux travaux de Drinfeld, de fournir les outils de géométrie analytique-rigide nécessaires à la réalisation du programme décrit par Carayol ([19]) autour de la correspondance de Langlands locale. Comme application, il prouve une conjecture de Deligne sur les cycles évanescents ([5]) qui est cruciale pour ce programme (voir plus loin ou l'article d'exposition de Carayol). Le lecteur pourra également consulter le compte-rendu de Berkovich à ICM 1998 ([8]), où Berkovich expose les idées sous-jacentes à sa théorie et ses motivations, ou bien encore lire l'exposé de Ducros au séminaire Bourbaki ([32]).

On considère les deux tours définies au paragraphe précédent :

$$
\begin{aligned}
& \mathrm{GL}_{d}(K) \circlearrowright \quad \mathcal{M}_{\mathcal{D} r, n}^{d} \quad \text { et } \quad \mathcal{M}_{\mathcal{L} \mathcal{T}, n}^{d} \quad \circlearrowleft D_{d}^{\times} \\
& \downarrow \circlearrowleft D_{d}^{\times} \quad \mathrm{GL}_{d}(K) \circlearrowright \downarrow \\
& \bigsqcup_{r \in \mathbb{Z}} \Omega^{d} \quad \bigsqcup_{r \in \mathbb{Z}} \stackrel{\circ}{B}^{d-1}
\end{aligned}
$$

Définition 1. - Les représentations locales fondamentales sont les espaces de cohomologie étale $\ell$-adique à support compact

$$
\mathcal{U}_{c, ?}^{i}=\underset{n}{\lim } \mathrm{H}_{c}^{i}\left(\left(\mathcal{M}_{?, n}^{d}\right)_{\bar{K}}, \overline{\mathbb{Q}}_{\ell}\right) \circlearrowleft \mathrm{GL}_{d}(K) \times D_{d}^{\times} \times W_{K}
$$

où $?=\mathcal{D}$ r ou $\mathcal{L} \mathcal{T}$ et où l'action sur la cohomologie résulte de l'action sur les tours décrite au paragraphe précédent.

Bien entendu, comme dans le formalisme $\ell$-adique traditionnel, on a

$$
\mathrm{H}_{c}^{i}\left(\left(\mathcal{M}_{?, n}^{d}\right)_{\bar{K}}, \overline{\mathbb{Q}}_{\ell}\right)={\underset{l}{m}}_{\lim _{c}} \mathrm{H}_{c}^{i}\left(\left(\mathcal{M}_{?, n}^{d}\right)_{\bar{K}}, \mathbb{Z} / \ell^{m} \mathbb{Z}\right) \otimes_{\mathbb{Z}_{\ell}} \overline{\mathbb{Q}}_{\ell}
$$


(plus exactement, Berkovich définit d'abord la cohomologie à support sur les ouverts distingués puis passe à la limite inductive).

Remarque 2. - Une définition alternative est la suivante : on considère

$$
\mathcal{U}_{\mathcal{L} \mathcal{T}}^{i}=\underset{n}{\lim } \operatorname{Ind}_{\left(\mathrm{GL}_{d}(K) \times D_{d}^{\times} \times W_{K}\right)^{1}}^{\mathrm{GL}_{d}(K) \times D^{\times} \times W_{K}} \mathrm{H}^{0}\left(\mathcal{M}_{\mathcal{L} \mathcal{T}, n}^{d,[0]} \otimes \bar{k}, \mathrm{R}^{i} \Psi_{\eta}^{\text {form }} \overline{\mathbb{Q}}_{\ell}\right)
$$

où $\Psi_{\eta}^{\text {form }}$ désigne le foncteur cycles évanescents de Berkovich. Ce foncteur calcule la cohomologie sans support, laquelle est duale de $\mathcal{U}_{c, \mathcal{L}} \mathcal{T}$ (dualité de Poincaré). Nous en dirons plus au paragraphe 5.2

Proposition 2.1. - Les représentations $\mathcal{U}_{c, ?}^{i}$ sont des représentations admissibles modulo le centre du groupe produit $\mathrm{GL}_{d}(K) \times D_{d}^{\times} \times W_{K}$.

Remarque 3. - Le centre de $\mathrm{GL}_{d}(K) \times D_{d}^{\times}$translate les composantes connexes $\mathcal{M}_{c, ?}^{d,[h]}, h \in \mathbb{Z}$, donc l'action ne peut etre admissible. C'est pourquoi dans le théorème 8 nous fixerons le caractère central $\chi:$ l'action est admissible sur le plus grand quotient $\mathcal{U}_{c, ?, \chi}^{i}$ de $\mathcal{U}_{c, \text { ? }}^{i}$ où le centre de $\mathrm{GL}_{d}(K)$ agit via $\chi$.

Ce résultat est essentiellement dû à Berkovich, qui a developpé une théorie des espaces analytiques munis d'une action de groupe (rappelée brievement dans l'appendice A de [47]).

Lemme 1 (Berkovich). - (i) Soit $\mathcal{U}$ un sous-ensemble ouvert distingué d'un K-espace analytique quasi-algébrique $X$. Alors les groupes de cohomologie à support compact $\mathrm{H}_{c}^{i}\left(\mathcal{U}_{\bar{K}}, \overline{\mathbb{Q}}_{\ell}\right)$ sont de dimension finie.

(ii) Si $X$ est de plus un $G$-espace (i.e. muni d'une action continue du groupe $G$ ) et $G$ contient un sous-groupe ouvert qui est un pro-p-groupe (avec $p \neq \ell)$, alors l'action de $G$ sur $\mathrm{H}_{c}^{i}\left(X_{\bar{K}}, \overline{\mathbb{Q}}_{\ell}\right)$ est lisse.

Le point (ii) démontre la lissité de l'action de $\mathrm{GL}_{d}(K)$ sur les espaces $\mathcal{U}_{c, \mathcal{D} r}^{i}$, celle de $D^{\times}$provient de la définition même des revêtements et l'action galoisienne est continue d'après un résultat général similaire de Berkovich. Par contre, le point (i) du lemme ne permet pas de démontrer l'admissibilité, mais seulement que la cohomologie à support est de type fini. En effet, on peut calculer la cohomologie par une suite spectrale correspondant à un recouvrement de Čech, qui fait intervenir un nombre fini d'induites compactes de représentations de la forme $\mathrm{H}_{c}^{i}\left(\mathcal{U}_{\bar{K}}, \overline{\mathbb{Q}}_{\ell}\right)$, pour des ouverts distingués $\mathcal{U}_{\bar{K}}$. Le résultat en découle et $\mathcal{U}_{c, \mathcal{D} r}^{i}$ est donc une représentation de longueur finie.

Du côté Lubin-Tate où les rôles de $D_{d}^{\times}$et $\mathrm{GL}_{d}(K)$ sont échangées, la situation est meilleure car $\mathcal{M}_{\mathcal{L} \mathcal{T}, n}^{d,[0]}$ est, en vertu du théorème d'uniformisation que 
nous énoncerons plus loin, un ouvert distingué d'un espace analytique quasialgébrique, si bien que $\mathrm{H}_{c}^{i}\left(\left(\mathcal{M}_{\mathcal{L} \mathcal{T}, n}^{d,[0]}\right)_{\bar{K}}, \overline{\mathbb{Q}}_{\ell}\right)$ est de dimension finie. L'admissibilité de l'action de $\mathrm{GL}_{d}(K) \times D_{d}^{\times}$sur $\mathcal{U}_{c, \mathcal{L} \mathcal{T}}^{i}$ en résulte directement, car $\mathcal{M}_{\mathcal{L} \mathcal{T}, n}^{d}$ est l'espace des invariants sous le sous-groupe de congruence principal $1+\varpi^{n} \mathrm{M}_{d}(K)$. De plus, la cohomologie sans support de $\mathcal{M}_{\mathcal{L} \mathcal{T}, n}^{d,[0]}$ (donc de $\mathcal{M}_{\mathcal{L} \mathcal{T}, n}^{d}$ par induction) a bien un sens, contrairement au côté Drinfeld.

En définitive, $\mathcal{U}_{c, \mathcal{D} r}^{i}$ est également admissible (modulo le centre), en vertu du théorème d'isomorphisme de Faltings-Fargues et son corollaire cohomologique, que nous allons maintenant exposer.

\section{L'isomorphisme de Faltings-Fargues}

Si l'article de Falting a ouvert la voie ([34]), il a fallu à Fargues quelques années de labeur afin de constuire les outils techniques nécessaires à rendre valides les arguments de loc. cit. Signalons également que Genestier et V. Lafforgue ont donné une seconde démonstration du résultat qui suit, plus simple mais valable uniquement en égale caractéristique et utilisant la théorie du module de coordonnées ([40]). Elle figure à la fin de l'ouvrage [39].

Le premier énoncé de [39] est le suivant :

Théorème 3 (Faltings-Fargues; [39] th. .0.1). - Il existe une "padification" $\widetilde{\widehat{\mathcal{M}}}_{\mathcal{L T}}$ de la tour de Lubin-Tate et un isomorphisme $\widetilde{\widehat{\mathcal{M}}}_{\mathcal{L T}} \stackrel{\sim}{\rightarrow} \widetilde{\widehat{\mathcal{M}}}_{\mathcal{D r}}$, où $\widetilde{\widehat{M}}_{\mathcal{D} r}$ est un certain éclatement formel admissible de $\widehat{\mathcal{M}}_{\mathcal{D} r}$. Cet isomorphisme est équivariant lorsque l'on munit $\widehat{\mathcal{M}}_{\mathcal{D} r}$ de l'action de $\mathrm{GL}_{d}(K) \times D_{d}^{\times}$ obtenue en tordant l'action naturelle par l'involution $(g, d) \mapsto\left({ }^{t} g^{-1}, d\right)$ de $\mathrm{GL}_{d}(K) \times D_{d}^{\times}$.

Apportons quelques éclaircissements, car donner un sens à l'isomorphisme est déjà un gros travail!

Remarque 4. - 1. Le schéma formel $\widehat{\Omega}$ est $\varpi$-adique : un idéal de définition de $\widehat{\Omega}$ est $(\pi)$ et il est localement de la forme $\operatorname{Spf}\left(\mathcal{O}_{K}\left\langle t_{1}, \ldots, t_{d}\right\rangle / \mathcal{I}\right)$ en tant que schéma formel (comme de tradition, $\mathcal{O}_{K}\left\langle t_{1}, \ldots, t_{d}\right\rangle$ désigne le complété $\varpi$-adique de $\mathcal{O}_{K}\left[t_{1}, \ldots, t_{d}\right]$, constitué des séries formelles restreintes). Les $\widetilde{\widehat{\mathcal{M}}}_{\mathcal{D} r, U}$ sont les normalisés de $\widehat{\Omega}$ dans $\mathcal{M}_{\mathcal{D} r, U}$ et $\widetilde{\widehat{\mathcal{M}}}_{\mathcal{D} r}=$ $\lim _{U} \widetilde{\widehat{\mathcal{M}}}_{\mathcal{D} r, U}$ est un schéma formel $\varpi$-adique qui constitue un modèle entier (formel) de la tour $\mathcal{M}_{\mathcal{D} r}$. 
2. On dispose également de modèle s entiers de la tour de Lubin-Tate : ils sont construits à partir de la notion de "base de Drinfeld" ([28]). Mais ils ne sont pas $\pi$-adiques car déjà en niveau zéro $\mathcal{O}_{K}\left[\left[t_{1}, \ldots, t_{d-1}\right]\right]$ ne l'est pas (un idéal de définition est $\left(\varpi, t_{1} \ldots, t_{d-1}\right)$ ). C'est pourquoi il faut $p$-adifier. Le modèle entier $\widetilde{\widehat{\mathcal{M}}}_{\mathcal{L T}}$ est construit en recollant des "cellules" indexées par l'immeuble de $\mathrm{PGL}_{d}$ (donc en utilisant les correspondances de Hecke), de façon à pouvoir comparer par la suite avec le côté Drinfeld.

3. L'isomorphisme n'est pas algébrique : il existe des $\bar{K}$-points qui s'envoient sur des $\widehat{\bar{K}} \backslash \bar{K}$-points. C'est d'ailleurs pour cela qu'il a fallu $\varpi$-adifier.

4. Les espaces $\mathcal{M}_{?}=\lim _{U} \mathcal{M}_{?, U}$ (pour ? = Dr et $\mathcal{L} \mathcal{T}$ ) ne sont pas des espaces rigides au sens classique du terme (ils ne le sont qu'en niveau fini). Le théorème permet de donner un sens à l'isomorphisme en recourant à de bons modèle s entiers. Signalons que Fargues a également développé une théorie des espaces rigides pour laquelle ces limites projectives ont un sens, indépendemment de l'existence de tels models.

Le second résultat de Fargues est une application cohomologique, qui est nouvelle par rapport aux travaux de Faltings. On peut paraphraser le théorème principal $13.2 \mathrm{du}$ chapitre IV de [39], étendu par le yoga habituel du cas des coefficients de torsion au cas des coefficients $\ell$-adiques, comme suit :

Théorème 4 (Fargues). - Soit † l'involution de $\mathrm{GL}_{d}(K)$ définie par $g \mapsto$ ${ }^{t} g^{-1}$; il y a un isomorphisme de $\mathrm{GL}_{d}(K) \times D^{\times} \times W_{K}$-modules lisse

$$
\mathrm{H}_{c}^{\cdot}\left(\mathcal{M}_{\mathcal{L} \mathcal{T}}^{d}\right) \simeq \mathrm{H}_{c}^{\cdot}\left(\mathcal{M}_{\mathcal{D} r}^{d}\right)^{\dagger}
$$

Remarque 5. - De façon précise, Fargues énonce un résultat d'équivalence de topos rigide-étale et obtient donc un résultat au niveau de la catégorie dérivée, dont a besoin Dat pour ses travaux que nous mentionnerons brièvement plus loin.

La constuction de l'isomorphisme entre les tours est extrêmement élaborée et les méthodes rigides employées très techniques, aussi est-il difficile d'expliquer en quelques mots pourquoi ça marche. Il est conseillé de commencer par comprendre l'isomorphisme au niveau des points (chapitre 2 de loc. cit.), ce qui ne nécessite pas de modèle entier particulier ni d'éclatement formel : on constate alors, suivant Fargues, que la théorie de Fontaine est au coeur de l'isomorphisme (périodes cristallines, etc.). On peut également consulter [36] pour une description de l'isomorphisme au niveau des "squelettes" des deux tours, ce qui permet de comprendre comment ces deux objets d'allure très dissemblable 
(rien qu'à les regarder en niveau zéro) puissent être en relation : méditer sur le dessin en page 2 de loc. cit. relatif au cas d'école $d=2$ et $K=\mathbb{Q}_{p}$.

\section{Les acteurs globaux}

Ce sont les variétés de Shimura dans le cas des corps de nombre (dont il est question dans [46] et dans le "survey" de Carayol) et les variétés modulaires de Drinfeld dans celui des corps de fonctions, que nous détaillons maintenant.

4.1. Les variétés modulaires de Drinfeld. - Commençons par un petit rappel historique : les premiers objets visibles dans la littérature sont les modules elliptiques de Drinfeld ([28]), qui apparaissent simultanément avec $\Omega^{d}$. En effet, Drinfeld montre que l'espace de module $M_{I}^{d}$ des modules elliptiques (rigidifiés par une structure de niveau $I$ et de degré $d$ ) constitue un analogue (pour $d=2$ ) des courbes modulaires $Y(N)$ : on obtient un résultat d'"uniformisation $p$-adique" semblable à la description usuelle de $Y(N)(\mathbb{C})$ comme quotient du demi-plan de Poincaré

$$
M_{I}^{d}\left(\widehat{F}_{\infty}\right) \simeq \mathrm{GL}_{d}(F) \backslash\left[\Omega^{d} \times \mathrm{GL}_{d}\left(\mathbb{A}^{\infty}\right)\right] / \mathrm{GL}_{d}\left(\mathcal{O}_{\infty}, I\right),
$$

où la place $\infty$ du corps global $F$ joue le rôle de la place archimédienne et où $\mathrm{GL}_{d}\left(\mathcal{O}_{\infty}, I\right)$ désigne le sous-groupe de $\mathrm{GL}_{d}\left(\mathcal{O}_{\mathbb{A}}\right)$ constituté des éléments qui sont congrus à 1 modulo $I$ (bien entendu, on a noté $\mathbb{A}$ les adèles de $F$ ). Il s'agit même d'un isomorphisme d'espaces rigides-analytiques. En calculant $\mathrm{H}^{1}:=\underset{I}{\lim } \mathrm{H}^{1}\left(\bar{M}_{I}^{2}\left(\widehat{F}_{\infty}\right), \overline{\mathbb{Q}}_{\ell}\right)$, Drinfeld réussit à établir un analogue de la théorie d'Eichler-Shimura : il attache une repésentation galoisienne $\ell$-adique de dimension 2 à toute représentation automorphe $\Pi$ de $G_{2}(\mathbb{A})$ telle que $\Pi_{\infty}$ est la Steinbeg $S t_{\infty}$. Cela lui permet de démontrer la correspondance de Langlands locale pour $d=2$ ([31]).

Drinfeld introduit alors de nouveaux objets, de nature plus géométrique (la définition d'un module elliptique est une définition algébrique élémentaire) : les faisceaux elliptiques et montre l'équivalence ("en dehors" de la place $\infty$ ) des deux notions ([30]; voir également [27]). Puis il définit les Stukas (ou Ffaisceaux, la place $\infty$ ne jouant plus de rôle particulier), ce qui lui permet de démontrer la correspondance de Langlands globale pour $d=2$.

Les variétés modulaires de Drinfeld ne sont pas propres, ce qui pose des problèmes ardus (compactification, formules des traces, etc.) qui sont difficiles à résoudre pour $d>2$. Une solution est apportée par Laumon, Rapoport et Stuhler à travers les $\mathcal{D}$-faisceaux elliptiques ([54]) qui sont donc des variantes 
compactes des faisceaux elliptiques de Drinfeld. Ces nouvelles variétés ne sont plus associées à $\mathrm{GL}_{d}$ mais au groupe multiplicatif d'un corps gauche $D$ de centre $F$, de dimension $d^{2}$ sur $F$ et déployé au-dessus de $\infty$. La correspondance de Langlands locale en égale caractéristique en résultera, en décomposant la cohomologie de l'espace de modules obtenu sous l'action des opérateurs de Hecke, de manière similaire à ce qu'à décrit Carayol pour les variétés de Shimura. Le lecteur pourra consulter [19] pour un compte-rendu agréable à lire de ces travaux (correspondance de Langlands locale uniquement).

Notations :

Soit $X / \mathbb{F}_{q}$ une courbe projective lisse, de corps de fonctions $F=\mathbb{F}_{q}(X)$ et soit $\mathcal{D}$ un faisceau d'ordres maximaux de $D$ et $\mathcal{R}$ le lieu de ramification de $D$. On se donne $\infty$,o deux places distinctes fixées tel que $D_{\infty}=\mathrm{M}_{d}\left(F_{\infty}\right)$. La place $\infty$ sera l'analogue de la place achimédienne et le complété $F_{o}$ jouera le rôle du corps local $K$ de la partie locale de cet exposé. Enfin, soit $I \subset X-\{\infty\}$ un sous-schéma fermé fini non vide.

La définition d'un $\mathcal{D}$-faisceau est essentiellement celle d'un diagramme de fibrés vectoriels sur la courbe $X$ :

Définition 2. - Un D-faisceau elliptique (de zéro $z: S \rightarrow X-\mathcal{R}-\{\infty\}$ et pôle $\infty$ ) défini sur un $\mathbb{F}_{q}$-schéma $S$ est la donnée d'un diagramme commutatif

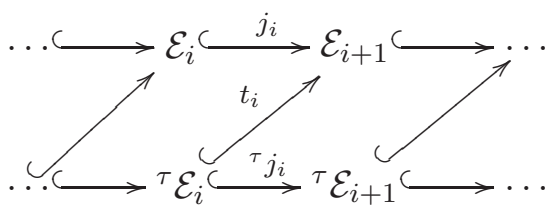

où les $\mathcal{E}_{i}$ sont des $\mathcal{D}_{X} \otimes \mathcal{O}_{S}$-modules à droite localement libres de rang $1,{ }^{\tau} \mathcal{E}_{i}=$ $\left(\operatorname{Id}_{X} \times \operatorname{Frob}_{S / \mathbb{F}_{q}}\right)^{*} \mathcal{E}_{i}$, et les conditions suivantes sont vérifiées :

(i) Périodicité : $\mathcal{E}_{i+d}=\mathcal{E}_{i}(\{\infty\} \times S)$

(ii) Pôle : $\mathcal{E}_{i+1 / j_{i}}\left(\mathcal{E}_{i}\right) \simeq\left(\Gamma_{\infty}\right)_{*} \mathcal{A}_{i}$, pour $\mathcal{A}_{i}$ un $\mathcal{O}_{S}$-module localement libre de rang $d$

(iii) Zéro: $\mathcal{E}_{i+1} / t_{i}\left({ }^{\tau} \mathcal{E}_{i}\right) \simeq\left(\Gamma_{z}\right)_{*} \mathcal{B}_{i}$, pour $\mathcal{B}_{i}$ un $\mathcal{O}_{S}$-module locallement libre de rang $d$

(iv) Normalisation : $\chi\left(\left.\mathcal{E}_{0}\right|_{X \times s}\right) \in[0, d[\quad$ pour tout point géométrique $s$

On retiendra qu'un $\mathcal{D}$-faisceau $\mathcal{E}$ possède un zéro et un pôle. Le morphisme zéro permet de voir l'espace de modules $\mathcal{E} \ell_{I}$ des $\mathcal{D}$-faisceaux elliptiques (munis d'une structure de niveau $I$ ) comme un schéma au-dessus de $X-\mathcal{R}-\{\infty\}$. Le pôle est quant à lui fixe (sans quoi on aboutit à la définition d'un $\mathcal{D}$-chtoucas 
de L. Lafforgue, qui parvient ainsi à démontrer la correspondance de Langlands globale pour $\mathrm{GL}_{d}, d>2$ ).

Théorème 5 ([54]). — Le schéma $\mathcal{E} \ell_{I}$ est projectif, lisse et purement de dimension relative $d-1$ au-dessus de $X-\mathcal{R}-\{\infty\}$.

Pour cela, Laumon, Rapoport et Stuhler vérifient (entre autres) le critère valuatif de propreté, en utilisant un théorème de réduction semi-stable développé par Drinfeld dans un travail sur la compactification des espaces de modules de Stukas.

Enfin, les $\mathcal{E} \ell \ell_{I}$ constituent un système projectif lorsque $I$ varie. Notant $\mathbb{A}^{\infty}$ les adèles "finies", on a une action à droite de $D^{\times}\left(\mathbb{A}^{\infty}\right)$ sur la limite projective $\varliminf_{I \notin \infty} \mathcal{E} \ell \ell_{I} \otimes \bar{F}_{o}$, définie via les opérateurs de Hecke.

4.2. Etude de la mauvaise réduction : les théorèmes d'uniformisation. - Il s'agit tout d'abord de prolonger le schéma $\mathcal{E} \ell \ell_{I}$ au-dessus de $\operatorname{Spec} \mathcal{O}_{o}$, dans les deux cas suivants :

(1) le cas où $o \in I$ (en dehors de $\mathcal{R}$ ), étudié par Boyer ([14]), qui va donner lieu à un théorème d'uniformisation faisant apparaêtre les espaces de LubinTate;

(2) le cas où $D_{o}$ est le corps gauche $D_{d}$ d'invariant $1 / d$, étudié par l'auteur de ce texte dans [47], où interviennent l'espace de Drinfeld (si $o \notin I$ ) et ses revêtements $\left(\operatorname{si~mult}_{o}(I)>0\right)$.

On autorise donc le zéro $z$ à intersecter le niveau et le lieu de ramification respectivement. Les propriétés des variétés de modules sont résumées ci-dessous :

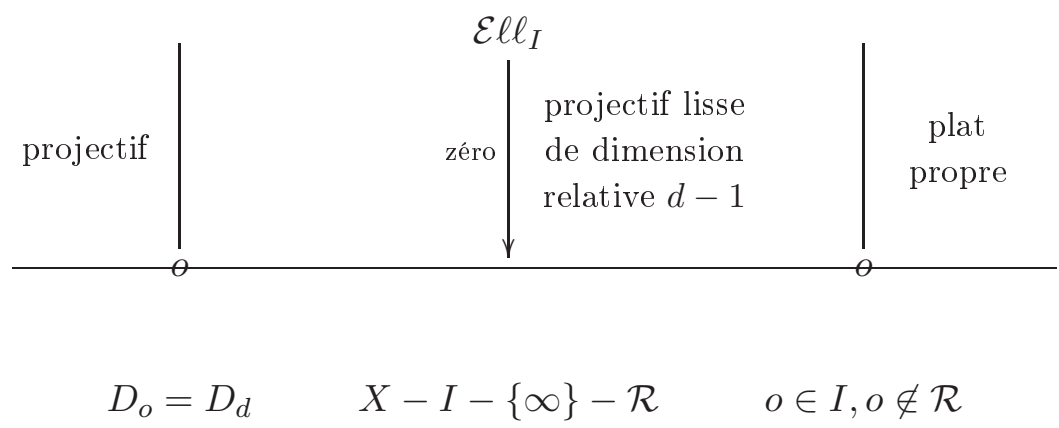

Pour que cela soit vrai, il faut imposer dans le cas (2) une condition supplémentaire, dite "condition spéciale" (sinon le schéma obtenu ne serait pas plat au-dessus de $\operatorname{Spec} \mathcal{O}_{o}$ ). A tout $\mathcal{D}$-faisceau elliptique est associé, par une construction due à Drinfeld, un $\mathcal{O}_{o^{-}}$-module $\varpi_{o^{-}}$divisible noté $G r_{o}(\mathcal{E})$, muni 
d'une action de $\mathcal{D}_{o}$, et l'on demande que ce soit un $\mathcal{D}_{o}$-module formel spécial, au sens du paragraphe 2.1. Noter également que les schémas de modules $\mathcal{E} \ell \ell_{I}$ ne sont plus lisses (leurs fibres spéciales sont très ramifiées au-dessus de Spec $k(o)$, comme on va le voir avec les théorèmes d'uniformisation).

Rappelons que les premiers résultats d'uniformisation $p$-adique remontent à Tate qui introduit dans [67] la notion d'espace analytique rigide en traitant du cas des courbes elliptiques à réduction multiplicative. Un peu plus tard, Mumford utilise le point de vue des schémas formels de Raynaud ([62]) pour montrer que les courbes de genres quelconques, en certaines places de mauvaise réduction, peuvent être uniformisées par des ouverts convenables du demiplan $p$-adique $\Omega_{\mathbb{Q}_{p}}^{2}=\mathbb{P}^{1}\left(\hat{\mathbb{Q}}_{p}\right)-\mathbb{P}^{1}\left(\mathbb{Q}_{p}\right)$ ([57]; voir aussi [61] et [58] pour une généralisation en dimension supérieure). Čerednik découvre alors ([21]) que tel est le cas de la courbe de Shimura $S$ associée à une algèbre de quaternions $\Delta$ indéfinie de centre $\mathbb{Q}$, en une place $p$ où $\Delta$ est ramifiée : $S \otimes \mathbb{Q}_{p}$ est la réunion de (formes tordues galoisiennes) de quotients à la Mumford de $\Omega_{\mathbb{Q}_{p}}^{2}$ par des sousgroupes de Schottky de $\mathrm{PGL}_{2}\left(\mathbb{Q}_{p}\right)$ (du moins, lorsque l'exposant en $p$ du niveau est maximal). Peu après, Drinfeld en donne une explication naturelle (voir [29] ou son exégèse [11] par Boutot et Carayol) en découvrant que $\widehat{\Omega}_{\mathbb{Q}_{p}}^{d} \hat{\otimes} \widehat{\mathbb{Z}}_{p}^{n r}$ est un espace de modules pour les $\mathcal{O}_{\Delta_{p}}$-modules formels (rigidifiés). L'uniformisation $p$-adique provient alors de ce qu'en la place $p$ considèrée, la courbe de Shimura paramètre des variétés abéliennes toutes isogènes dont le groupe $p$-divisible est de ce type. Depuis, l'uniformisation $p$-adique des variétés de Shimura a été développée par de nombreux auteurs ([18], [59], [60], [68]; voir également l'article d'exposition [12]).

Nous allons maintenant expliciter les théorèmes d'uniformisation dans les deux cas qui nous concernent. Par rapport à ces rappels historiques, le cas $D_{o}=D_{d}$ est l'analogue de l'uniformisation de Čerednik-Drinfeld. Le cas de Boyer est l'analogue du résultat obtenu par Carayol pour certaines variétés de Shimura ([18]).

4.2.1. Le cas $D_{o}=D_{d}$. - Les raisons de l'uniformisation sont les mêmes que celles évoquées plus haut : d'une part, tous les $\mathcal{D}$-faisceau elliptique $\mathcal{E}$ définis sur $\overline{k(o)}$ sont isogènes. Leurs fibres génériques sont donc isomorphes et l'on montre que l'algèbre $\bar{D}$ des endomorphismes d'une telle fibre générique s'identifie à $D$ en dehors des places $\infty, o$ et vérifie $\bar{D}_{\infty}=D_{d}$ tandis que $\bar{D}_{o}$ est déployé. Le groupe $\varpi_{o}$-divisible $G r_{o}(\mathcal{E})$ associé à $\mathcal{E}$ est un $\mathcal{D}_{o}$-module spécial. Notant $\Phi$ l'unique (à isogénie près) $\mathcal{D}_{o}$-module formel spécial sur $\overline{k(o)}$, l'ensemble des 
algébrisations de $\Phi$, c'est-à-dire des $\mathcal{D}$-faisceaux elliptiques sur $\overline{k(o)}$ munis d'un isomorphisme $\Phi \simeq G r_{o}(\mathcal{E})$, est en bijection avec l'ensemble de doubles-classes

$$
Z_{I}=\bar{D}^{\times}(F) \backslash \bar{D}^{\times}\left(\mathbb{A}^{\infty}\right) / K_{I}^{\infty, o},
$$

où l'on a noté $K_{I}^{\infty, o}$ le sous-groupe compact ouvert de $\bar{D}^{\times}\left(\mathbb{A}^{\infty}\right)$ constitué des éléments qui s'envoient sur 1 modulo $I$. On utilise alors la description modulaire de l'espace $\widehat{\Omega} \frac{d}{k(o)}$ pour construire une application $\widehat{\Omega} \frac{d}{k(o)} \times Z_{I} \rightarrow \mathcal{E} \ell \ell_{I} \otimes \overline{k(o)}$. Rentre ensuite en scène le second ingrédient essentiel, à savoir l'analogue du théorème de Serre et Tate qui dit que déformer un $\mathcal{D}$-faisceau elliptique $\mathcal{E}$ revient à déformer son groupe $\varpi_{o}$-divisible $G r_{o}(\mathcal{E})$.

On démontre ainsi :

Théorème 6 (Hausberger; [47] 8.2). - (a) Si o $\notin$ I alors il existe un isomorphisme de $\mathcal{O}_{o}$-schémas formels

$$
{\widehat{\mathcal{E} \ell \ell_{I}}}_{\left\{s_{o}\right\}} \simeq\left[\left(\widehat{\Omega}^{d} \hat{\otimes} \widehat{\mathcal{O}}_{o}^{n r}\right) \times Z_{I}\right] / \mathrm{GL}_{d}\left(F_{o}\right),
$$

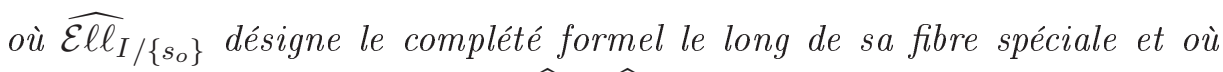
l'action à droite de $\mathrm{GL}_{d}\left(F_{o}\right)$ sur $\widehat{\Omega}^{d} \hat{\otimes}^{\text {O्O }}{ }^{n r}$ est l'action décrite au paragraphe 2 composée avec l'involution $g \mapsto{ }^{t} g^{-1}$.

(b) Si par contre mult $_{o}(I)=n$ alors il existe un isomorphisme d'espaces rigides-analytiques sur $F_{O}$ :

$$
\left(\mathcal{E} \ell \ell_{I}\right)^{a n} \simeq\left[\Sigma_{n}^{d} \times Z_{I^{o}}\right] / \mathrm{GL}_{d}\left(F_{o}\right) .
$$

Ces isomorphismes sont compatibles, lorsque I varie (i.e. $n$ et $I^{o}$ varient), aux opérations de projection, et l'isomorphisme ainsi obtenu entre les deux systèmes projectifs est équivariant pour l'action du groupe $D^{\times}\left(\mathbb{A}^{\infty}\right) \simeq$ $\bar{D}^{\times}\left(\mathbb{A}^{\infty, o}\right) \times D_{o}^{\times}$.

Remarque 6. - 1 . Le $\mathcal{D}_{o}$-module formel $G r_{o}(\mathcal{E})$ ) provient en fait de la fibre en $o$ du $\mathcal{D}$-faisceau elliptique $\mathcal{E}$, qui est un $\mathcal{D}_{o^{-}}$-module de coordonnées. Or la théorie du module de coordonnées, qui à un tel objet fait correspondre un module formel (et réciproquement), est une théorie de Dieudonné contravariante. L'identification de $\bar{D}$ avec l'algèbre d'endomorphismes de la fibre générique de $\mathcal{E}$ induit ainsi un isomorphisme $\mathrm{M}_{d}\left(F_{o}\right)=\bar{D}_{o} \simeq \operatorname{End}_{\mathcal{D}_{o}}^{0}(\Phi)$ tel que $g$ correspond à la quasi-isogénie ${ }^{t} g^{-1}$. Cela explique la présence de l'involution dans l'énoncé du théorème.

2. L'uniformisation (b) est uniquement rigide-analytique car il n'y a pas de modèle formel connu des revêtements de Drinfeld. C'est essentiellement une conséquence formelle de (a) : l'isomorphisme décrit se relève 
au niveau des $\mathcal{D}_{o^{-}}$-modules formels spéciaux portés par les deux membres (l'un est associé au $\mathcal{D}$-faisceau elliptique universel au-dessus de ${\widehat{\mathcal{E} \ell \ell_{I}}}_{/\left\{s_{o}\right\}}$ par la construction $G r_{o}$ de Drinfeld et l'autre provient de la description modulaire de $\left.\widehat{\Omega}^{d} \hat{\otimes} \widehat{\mathcal{O}}^{n r}\right)$. Il suffit alors de considérer la $\varpi_{o}^{n}$-torsion.

3. L'isomorphisme obtenu est un isomorphisme d'espaces analytiques sur $F_{o}$ et non sur $F_{o}^{n r}$ : il y a des torsions galoisiennes et le quotient de droite est en définitive une union disjointe finie de tordues galoisiennes de quotients $\Sigma_{n}^{d} / \Gamma$ pour des sous-groupes de congruences $\Gamma \subset \mathrm{PGL}_{d}(K)$.

4. Une formulation alternative est la suivante : il existe un isomorphisme d'espaces rigides-analytiques sur $\widehat{F}_{o}^{n r}$ :

$$
\left(\mathcal{E} \ell \ell_{I}\right)^{a n} \hat{\otimes} \widehat{F}_{o}^{n r} \simeq\left[\mathcal{M}_{\mathcal{D} r, n}^{d} \times Z_{I^{o}}\right] / \mathrm{GL}_{d}\left(F_{o}\right)
$$

compatible à l'action du Frobenius Frob ${ }_{o}$ et celle (par correspondances) de $D^{\times}\left(\mathbb{A}^{\infty}\right) \simeq \bar{D}^{\times}\left(\mathbb{A}^{\infty, o}\right) \times D_{o}^{\times}$.

4.2.2. Le cas $o \in I$. - La géométrie est beaucoup plus riche dans le cas étudié par Boyer. En effet, la fibre spéciale $\mathcal{E} \ell \ell_{I, s_{o}}$ au-dessus de $s_{o}=\operatorname{Spec} k(o)$ est stratifiée par des sous-schémas localement fermés $\mathcal{E} \ell \ell_{I, s_{o}}^{=h}, 1 \leq h \leq d$, de pures dimensions $d-h$, caractérisés par la propriété qu'en tout point géométrique $\bar{z}_{o}$ de $\mathcal{E} \ell \ell_{I, s_{o}}^{=h}$ le $\mathcal{O}_{o}$-module $\varpi_{o}$-divisible $G=G r_{o}(\mathcal{E})$ se décompose en parties étale et connexe :

$$
1 \rightarrow G_{\bar{z}_{o}}^{e ́ t} \rightarrow G_{\bar{z}_{o}} \rightarrow G_{\bar{z}_{o}}^{c} \rightarrow 1
$$

avec $G_{\bar{z}_{o}}^{c}$ de hauteur $h$ (et donc $G_{\bar{z}_{o}}^{e ́ t}$ de hauteur $d-h$ ). Cela contraste avec le cas précédent où l'action de l'algèbre à division rigidifiait tout.

On rappelle que $\Phi_{h}$ désigne l'unique (à isogénie près) $\mathcal{O}_{o}$-module formel de dimension 1 et hauteur $h$. On a vu que le foncteur des déformations de $\Phi_{h}$ par quasi-isogénie est représentable par le schéma formel $\widehat{\mathcal{M}}_{\mathcal{L} \mathcal{T}, 0}^{h}$. La composante connexe correspondant aux quasi-isogénies de hauteur zéro vérifie $\widehat{\mathcal{M}}_{\mathcal{L} \mathcal{T}, 0}^{h,[0]} \simeq \operatorname{Spf} \widehat{\mathcal{O}}_{o}^{n r}\left[\left[t_{1}, \ldots, t_{h-1}\right]\right]$. En rajoutant une structure de niveau $n$, on obtient $\widehat{\mathcal{M}}_{\mathcal{L} T, n}^{h,[0]} \simeq \operatorname{Spf} \operatorname{Def}_{n}^{h}$ (consulter [28] $\S 4$ pour les équations).

Notons $F_{o} / \mathcal{O}_{o}$ le $\mathcal{O}_{o}$-module $\varpi_{o^{-}}$-divisible étale de hauteur 1 sur $\overline{k(o)}$ et $\Phi_{h, h^{\prime}}=$ $\Phi_{h} \times\left(F_{o} / \mathcal{O}_{o}\right)^{h^{\prime}}$. Le groupe des quasi-isogénies de $\Phi_{h, h^{\prime}}$ s'identifie à $D_{h}^{\times} \times$ $\mathrm{GL}_{h^{\prime}}\left(F_{o}\right)$, où $D_{h}$ désigne l'algèbre à division sur $F_{o}$ d'invariant $1 / h$. On obtient encore un foncteur représentable et la partie étale rajoute $h^{\prime}$ variables à l'anneau de séries formelles.

Boyer démontre alors un analogue du théorème de Serre et Tate : déformer un $\mathcal{D}$-faisceau elliptique $\mathcal{E}$ revient à déformer le $\mathcal{O}_{o}$-module $\varpi_{o}$-divisible $G r_{o}(\mathcal{E})$, lequel est de la forme $\Phi_{h, d-h}$ au sein de la strate $\mathcal{E} \ell_{I, s_{o}}^{=}$. En définitive, le 
complété formel en un point géométrique $\bar{z}_{o}$ de $\mathcal{E} \ell \ell_{I, s_{o}}^{\bar{h}}$ s'exprime

$$
\widehat{\mathcal{E} \ell \ell}_{I /\left\{\bar{z}_{o}\right\}} \simeq \operatorname{Spf}\left(\operatorname{Def}_{n}^{h}\left[\left[t_{1}, \ldots, t_{d-h}\right]\right]\right)
$$

où $n=\operatorname{mult}_{o}(I)$.

Regardons maintenant ce qui se passe lorsque $h=d$ (par analogie avec le cas des courbes elliptiques, cela correspond au lieu supersingulier). Alors $G r_{o}(\mathcal{E})$ est infinitésimal et l'on démontre que $\mathcal{E} \ell \ell_{I, s_{o}}^{=d}(\overline{k(o)})$ correspond à une seule classe d'isogénie (de $\mathcal{D}$-faisceax elliptiques). L'algèbre $\bar{D}$ des endomorphismes de la fibre générique de ces $\mathcal{D}$-faisceaux est telle qu'elle s'identifie à $D$ en dehors des places $\infty, o$ et vérifie $\bar{D}_{o}=D_{d}, \bar{D}_{\infty}=D_{d}^{o p p}=D_{-d}$. Comme plus haut, il en résulte un paramétrage de $\mathcal{E} \ell \ell_{I, s_{o}}^{=d}(\overline{k(o)})$ par les doubles classes

$$
Z_{I}=\bar{D}^{\times}(F) \backslash \bar{D}^{\times}\left(\mathbb{A}^{\infty}\right) / K_{I}^{\infty, o}
$$

(à la différence près que l'algèbre $\bar{D}$ n'est pas la même).

Combinant ce fait avec l'analogue du résultat de Serre-Tate, Boyer démontre :

Théorème 7 (Boyer; [14]). - Il existe un isomorphisme de schémas formels sur $\widehat{\mathcal{O}}_{o}^{n r}$ :

$$
{\widehat{\mathcal{E} \ell \ell_{I}}}_{\left\{s_{o} ;=d\right\}} \simeq\left[\widehat{\widehat{\mathcal{M}}}_{\mathcal{L} \mathcal{T}, n} \times Z_{I^{o}}\right] / \bar{D}_{o}^{\times}
$$

compatible à l'action du Frobenius Frob $_{o}$ et celle (par correspondances) de $D^{\times}\left(\mathbb{A}^{\infty}\right) \simeq \bar{D}^{\times}\left(\mathbb{A}^{\infty, o}\right) \times D_{o}^{\times}$, où $n=\operatorname{mult}_{o}(I)$ et ${\widehat{\mathcal{E} \ell \ell_{I}}}_{\left\{s_{o} ;=d\right\}}$ désigne le compété formel de $\mathcal{E} \ell_{I} \otimes_{\mathcal{O}_{o}} \widehat{\mathcal{O}}_{o}^{n r}$ le long de $\mathcal{E} \ell \ell_{I, s_{o}}^{=} \otimes_{k(o)} \overline{k(o)}$.

Remarque 7. - 1. Nous avons légèrement reformulé le résultat de loc. cit. (prop. 14.2) afin de mettre en avant le parallèle entre les deux cas d'uniformisation.

2. La notation $\widehat{\mathcal{M}}_{\mathcal{L} \mathcal{T}, n}^{d}$ signifie que l'action de $\mathrm{GL}_{d}\left(F_{o}\right) \times \bar{D}_{o}^{\times}$est modifiée par rapport à celle définie au paragraphe 2 afin de tenir compte du fait que la construction du $\mathcal{O}_{o}$-module $\varpi_{o}$-divisible $G r_{o}(\mathcal{E})$ passe par la théorie du module de coordonnées.

\section{Les suites spectrales}

Elles relient les représentations locales fondamentales :

$$
\mathcal{U}_{c, \mathcal{D} r}^{i}, \mathcal{U}_{\mathcal{L} \mathcal{T}}^{i} \circlearrowleft \mathrm{GL}_{d}\left(F_{o}\right) \times D_{o}^{\times} \times W_{F_{o}}
$$

à la représentation globale :

$$
H_{o}^{i}:=\underset{I \not \supset \infty}{\lim _{I}} H^{i}\left(\mathcal{E} \ell \ell_{I} \otimes \bar{F}_{o}, \overline{\mathbb{Q}}_{\ell}\right) \circlearrowleft D^{\times}\left(\mathbb{A}^{\infty}\right) \times W_{F_{o}}
$$


(action définie à l'aide des opérateurs de Hecke).

\subsection{La suite spectrale de Hochschild-Serre. -}

Proposition 5.1 (Harris-Hausberger ; [47] cor. 10.7)

Il existe une suite spectrale $\bar{D}^{\times}\left(\mathbb{A}^{\infty, o}\right) \times D_{o}^{\times} \times W_{F_{o}} \simeq D^{\times}\left(\mathbb{A}^{\infty}\right) \times W_{F_{o}}$ équivariante :

$$
\mathrm{E}_{2}^{i, j}=\operatorname{Ext}_{\mathrm{GL}_{d}\left(F_{o}\right)-\text { mod. lisse }}^{i}\left(\mathcal{U}_{c, \mathcal{D} r}^{2(d-1)-j, \dagger}(d-1), \mathcal{A} \frac{\infty}{D}\right) \Longrightarrow H_{o}^{i+j},
$$

où $\mathcal{A} \frac{\infty}{D}$ désigne l'espace des formes automorphes sur $\bar{D}$ triviales à l'infini et $\dagger$ l'involution de $\mathrm{GL}_{d}\left(F_{o}\right)$ définie par $g \mapsto^{t} g^{-1}$.

Via le théorème d'uniformisation, il s'agit de relier la cohomologie de $\left(\mathcal{M}_{\mathcal{D} r, n}^{d}\right)_{\bar{F}_{o}}$ à celle du quotient $\left[\left(\mathcal{M}_{\mathcal{D} r, n}^{d}\right)_{\bar{F}_{o}} \times Z_{I^{o}}\right] / \mathrm{GL}_{d}\left(F_{o}\right) \simeq\left(\mathcal{E} \ell \ell_{I}\right)_{\bar{F}_{o}}$, d'où la terminologie. S'inspirant du travail réalisé par Harris dans le cas des corps de nombres ([41]), cette suite spectrale a été construite "à la main", en utilisant des recouvrements de Čech (sur des ouverts distingués où le revêtement est trivialisable car le groupe agit librement) et la théorie de Berkvich des espaces analytiques munis d'une action de groupe. En effet, il n'existait pas de résultat général valable pour les espaces considérés. Depuis, Fargues a écrit une suite spectrale de Hochschild-Serre qui s'applique à tous les résultats d'uniformisation de ce type connus ([35] 4.5.2).

5.2. La suite spectrale des cycles évanescents. - Soit $X$ un $\operatorname{Spf} \widehat{\mathcal{O}}_{o}^{n r}$ schéma formel (spécial au sens de [5]). Le foncteur $\mathrm{R} \Psi_{\eta_{o}}^{\text {form }}$ associe à un faisceau étale sur la fibre générique $X_{\bar{\eta}_{o}}$ de $X$ (au sens de Raynaud) un complexe de faisceaux étales constructibles sur la fibre spéciale $X_{\bar{s}_{o}}$. Si on applique cette construction à $X=\widehat{\mathcal{M}}_{\mathcal{L} T}^{d,[0]}$ dont la fibre spéciale est réduite à un point, on obtient des faisceaux $\mathrm{R}^{i} \Psi_{\eta_{o}}^{\text {form }} \overline{\mathbb{Q}}_{\ell}$ tels que

$$
\mathrm{H}^{0}\left(\mathcal{M}_{\mathcal{L} \mathcal{T}, n}^{d,[0]} \otimes \overline{k(o)}, \mathrm{R}^{i} \Psi_{\eta_{o}}^{\text {form }} \overline{\mathbb{Q}}_{\ell}\right)=\mathrm{H}^{i}\left(\left(\mathcal{M}_{\mathcal{L} \mathcal{T}, n}^{d,[0]}\right)_{\bar{F}_{o}}, \overline{\mathbb{Q}}_{\ell}\right),
$$

de sorte que la représentation locale fondamentale (version sans support) n'est autre que

$$
\mathcal{U}_{\mathcal{L} \mathcal{T}}^{i}=\underset{n}{\lim } \operatorname{Ind}_{\left(\mathrm{GL}_{d}(K) \times D_{d}^{\times} \times W_{K}\right)^{1}}^{\mathrm{GL}_{d}(K) \times D_{d}^{\times} \times W_{K}} \mathrm{H}^{0}\left(\mathcal{M}_{\mathcal{L} \mathcal{T}, n}^{d,[0]} \otimes \overline{k(o)}, \mathrm{R}^{i} \Psi_{\eta}^{\text {form }} \overline{\mathbb{Q}}_{\ell}\right) .
$$

D'une façon générale, si $Z \rightarrow \operatorname{Spec} \widehat{\mathcal{O}}_{o}^{n r}$ est un schéma et $\bar{x}$ un point géométrique de la fibre spéciale $Z_{s}$, on peut considérer d'une part le complexe des cycles évanescents algébriques $\mathrm{R} \Psi_{\eta_{o}} \overline{\mathbb{Q}}_{\ell}$ sur $Z_{s}$, et d'autre part celui des cycles 
évanescents formels $\mathrm{R} \Psi_{\eta_{o}}^{\text {form }} \overline{\mathbb{Q}}_{\ell}$ associés au complété formel $X=\widehat{Z}_{/\{\bar{x}\}}$ de $Z$ en $\bar{x}$. Berkovich démontre le théorème de comparaison ([5]) :

$$
\left(\mathrm{R} \Psi_{\eta_{o}} \overline{\mathbb{Q}}_{\ell}\right)_{\bar{x}} \simeq \mathrm{R} \Psi_{\eta_{o}}^{\text {form }} \overline{\mathbb{Q}}_{\ell} .
$$

Prenant $Z=\mathcal{E} \ell \ell_{I} \otimes_{\mathcal{O}_{o}} \widehat{\mathcal{O}}_{o}^{n r}$ et $\bar{z}_{o}$ un point supersingulier, il résulte alors du théorème de Serre-Tate (voir paragraphe 4.2.2) un isomorphisme

$$
\left(\mathrm{R} \Psi_{\eta_{o}} \overline{\mathbb{Q}}_{\ell}\right)_{\bar{z}_{o}} \simeq \mathrm{R} \Psi_{\eta_{o}}^{\text {form }} \overline{\mathbb{Q}}_{\ell},
$$

où les cycles évanescents formels sont ceux du début de ce paragraphe qui calculent la cohomologie de $\mathcal{M}_{\mathcal{L} \text { T, }, n}^{d,[0]}$. Pour la strate supersingulière toute entière, uniformisée par le théorème 7 , on trouve

$\mathrm{H}^{0}\left(\mathcal{E} \ell \ell_{\bar{I}, s_{o}}^{=d} \otimes \overline{k(o)}, \mathrm{R}^{i} \Psi_{\eta_{o}} \overline{\mathbb{Q}}_{\ell}\right) \simeq\left[\mathrm{H}^{0}\left(Z_{I^{o}}, \overline{\mathbb{Q}}_{\ell}\right) \otimes \mathrm{H}^{0}\left(\widetilde{\mathcal{M}_{\mathcal{L} T, n}^{d}} \otimes \overline{k(o)}, \mathrm{R}^{i} \Psi_{\eta_{o}}^{f o r m} \overline{\mathbb{Q}}_{\ell}\right)\right]^{\bar{D}_{o}}$, où $\operatorname{mult}_{o}(I)=n$. Passant à la limite sur $I$, on obtient ainsi la description suivantes des cycles évanescents supersinguliers :

Proposition 5.2 (Boyer [14]). - Il existe un isomorphisme équivariant pour l'action de $D^{\times}\left(\mathbb{A}^{\infty}\right) \times W_{F_{o}} \simeq \bar{D}^{\times}\left(\mathbb{A}^{\infty, o}\right) \times D_{o}^{\times} \times W_{F_{o}}$

$$
\underset{I \not \supset \infty}{\lim _{I \not \infty}} \mathrm{H}^{0}\left(\mathcal{E} \ell \ell_{I, s_{o}}^{=d} \otimes \overline{k(o)}, \mathrm{R}^{i} \Psi_{\eta_{o}} \overline{\mathbb{Q}}_{\ell}\right) \simeq \operatorname{Hom}_{\bar{D}_{o}^{\times}}\left(\check{\mathcal{A}}_{\bar{D}}^{\infty}, \widetilde{\mathcal{U}_{\mathcal{L T}}^{i}}\right),
$$

où $\mathcal{A} \frac{\infty}{D}$ désigne l'espace des formes automorphes sur $\bar{D}$ triviales à l'infini, $\check{\mathcal{A}}_{\bar{D}}^{\infty}$ sa contragrédiente et $\widetilde{\mathcal{U}_{\mathcal{L T}}^{i}}$ indique une légère modification de l'action (voir remarque 7 2.).

Enfin, la cohomologie des cycles évanescents est reliée à celle de la variété globale par la suite spectrale suivante :

Proposition 5.3. - Il existe une suite spectrale $D^{\times}\left(\mathbb{A}^{\infty}\right) \times W_{F_{o}}$-équivariante :

$$
\mathrm{E}_{2}^{i, j}=\underset{I \not \supset \infty}{\lim _{c}} \mathrm{H}_{c}^{i}\left(\mathcal{E} \ell \ell_{I, s_{o}} \otimes_{k(o)} \overline{k(o)}, \mathrm{R}^{j} \Psi_{\eta_{o}} \overline{\mathbb{Q}}_{\ell}\right) \Longrightarrow H_{o}^{i+j}
$$

\section{Réalisations géométriques des correspondences}

\subsection{Enoncé du théorème. -}

Théorème 8 (Boyer). - Soit $\chi$ un caractère de $K^{\times}$d'ordre fini; notant avec un indice $\chi$ le plus grand quotient où le centre de $\mathrm{GL}_{d}(K)$ (resp. celui 
de $\left.D^{\times}\right)$agit par $\chi\left(\right.$ resp. $\left.\chi^{-1}\right)$, on a la décomposition suivante :

$$
\begin{aligned}
& \left(\mathcal{U}_{c, \mathcal{D} r, \chi}^{d-1+i}\right)^{F r-s s}=\left(\text { resp. }\left(\mathcal{U}_{c, \mathcal{L}}^{d-1+i},\right)^{F r-s s}=\right) \\
& \left\{\begin{array}{lll}
\underset{\substack{s \geq i+1 \\
s \mid d}}{\bigoplus} \underset{\substack{\mathcal{A}_{\frac{d}{s}, \chi}^{0} \\
\text { (K) }}}{\bigoplus} & S_{i, s}(\pi) \otimes \mathrm{JL}\left(S t_{s}(\pi)\right) \otimes \mathrm{L}(\pi)\left(-i-\frac{d-s}{2}\right) & \text { si } 0 \leq i \leq d-1, \\
0 & \left(\text { resp. } S_{i, s}(\pi) \otimes \mathrm{JL}\left(S t_{s}\left(\pi^{\vee}\right)\right) \otimes \mathrm{L}\left(\pi^{\vee}\right)\left(-i-\frac{d-s}{2}\right)\right) & \text { sinon, }
\end{array}\right.
\end{aligned}
$$

où $S t_{s}(\pi)$ (resp. Speh ${ }_{i}(\pi)$ désigne la représentation de Steinberg (resp. de Speh) généralisée et $S_{i, s}(\pi)$ est l'unique sous-module irréductible de l'induite normalisée $\operatorname{Speh}_{i}(\pi)||^{\frac{i-s}{2}} \times S t_{s-i}(\pi)||^{\frac{i}{2}}(0 \leq i \leq s-1)$

Donnons quelques éclaircissements et interprétations :

1. Toutes ces représentations sont des sous-quotients de $\pi||^{\frac{1-s}{2}} \times \cdots \times \pi||^{\frac{s-1}{2}}$. On parle de représentations elliptiques. La Steinberg (resp. la représentaion de Speh) généralisée $S t_{s}(\pi)$ (resp. $S p e h_{i}(\pi)$ ) est l'unique quotient (resp. sous-espace) iréductible de cette induite. Il résulte de la théorie de Bernstein et Zelevinsky que l'induite $\operatorname{Speh}_{i}(\pi)||^{\frac{i-s}{2}} \times S t_{s-i}(\pi)||^{\frac{i}{2}}$ est de longueur deux. Par rapport à la classification, son unique sousmodule irréductible $S_{i, s}(\pi)$ correspond à l'orientation du graphe de sommets $s_{i}=\left(\pi||^{i-1+\frac{1-s}{2}}\right)_{1 \leq i \leq s}$ et d'arêtes $\left(s_{i}, s_{i+1}\right)$ donné par

$$
\stackrel{s_{1}}{\bullet} \rightarrow \stackrel{s_{2}}{\bullet} \rightarrow \ldots \rightarrow \stackrel{s_{i}}{\rightarrow} \rightarrow \stackrel{s_{i+1}}{\bullet} \leftarrow \ldots \leftarrow \stackrel{s_{s}}{\bullet}
$$

(et son unique quotient irréductible à l'orientation

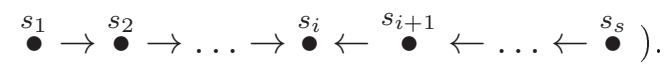

Il correspond à $S_{i, s}(\pi)$ par la correspondance de Langlands locale la représentation

$\mathrm{L}(\pi)\left(\frac{1-s}{2}\right) \oplus \cdots \oplus \mathrm{L}(\pi)\left(\frac{s-1}{2}\right)$ où la matrice de la monodromie $N$ se termine par son unique bloc de Jordan non trivial qui est de taille $s-i$.

2. Ce résultat a été conjecturé par Carayol pour $i=0$ (et démontré dans le cas $d=2$, voir [18] et [17], le cas $d=1$ étant équivalent à la théorie de Lubin-Tate [55], [56]) et par Harris en dehors du degré médian (notes non publiées). Pour la partie supercuspidale de la cohomologie, la conjecture était connue sous le nom de "conjecture de Deligne-Carayol" dans le cas Lubin-Tate et "conjecture de Drinfeld-Carayol" dans le cas des revêtements de Drinfeld. Pendant longtemps, les seuls résultats connus sur la partie non supercuspidale de la cohomologie était dûs à Schneider et Stuhler ([65]) qui explicitent la cohomologie (sans support) de l'espace 
de base $\Omega^{d}$. Ce sont en partie ces résultats qui inspirèrent à Harris sa conjecture.

3. Un grand soin doit être accordé à la normalisation des diverses actions pour que le théorème soit correct tel qu'énoncé précédemment. De plus, les différents auteurs prennent, selon le contexte, la cohomologie avec ou sans support. Une autre source potentielle d'erreur est que la partie galoisienne de la cohomologie qu'isolent Harris et Taylor fait apparaêtre une contragrédiente (cas des corps $p$-adiques) qui ne figure pas dans l'énoncé de Laumon, Rapoport et Stuhler. Cela "compense" en fait la contragrédiente introduite en égale caractéristique par la construction légèrement différente du groupe formel sous-jacent. En définitive, différentes variantes existent dans la littérature et l'auteur de ce texte espére avoir donné un énoncé cohérent en reproduisant ainsi la conjecture 9.6 de [47].

4. Le théorème décrit donc la Frob-semi-simplifiée de la représentation locale fondamentale $\mathcal{U}_{c}^{i}$. L'action de $\mathrm{GL}_{d}(K) \times D_{d}^{\times} \times I_{K}$ sur la cohomologie est semi-simple mais Boyer travaille à Frob-semi-simplification près.

5. Les séries discrètes (c'est-à-dire les Steinberg généralisées) sont concentrées en degré médian $d-1$ où l'on obtient une somme de termes de la forme $\left.S t_{s}(\pi) \otimes \mathrm{JL}\left(S t_{s}\left(\pi^{?}\right)\right) \otimes \mathrm{L}\left(\pi^{?}\right)\left(-i-\frac{d-s}{2}\right)\right)$. La correspondance de Jacquet-Langlands est donc réalisée (à contragrédiente près dans le cas Lubin-Tate), mais la correspondance de Langlands locale l'est uniquement pour les supercuspidales $(s=1)$.

Enfin, sans mener de calcul, expliquons pourquoi la cohomologie à support est concentrée en degrés compris entre $d-1$ et $2(d-1)$. Cela est possible du côté de Drinfeld, grâce à des résultats de dimension cohomologique dûs à Berkovich, valables pour une classe d'espaces analytiques incluant les revêtements de Drinfeld.

Comme $\mathcal{M}_{\mathcal{D} r}^{d}$ est de dimension $d-1$, la cohomologie avec support est en degrés au plus 2(d-1) (cf. [47] Annexe A). Pour l'autre inégalité, on utilise les résultats de [7], qui s'appliquent à $\mathcal{M}_{\mathcal{D} r, K_{n}}^{d,[0]}=\Sigma_{n}^{d}$ ( $c f$. introduction de loc. cit. ) : les revêtements de Drinfeld peuvent s'écrire comme une union croissante de sous-domaines analytiques compacts quasi-affines $V_{m}$ (au sens de [7] déf. 5.5). D'après le corollaire 6.2 du théorème 6.1 de loc. cit. , la cohomologie $\mathrm{H}_{c}\left(V_{m} \hat{\otimes} \widehat{\bar{K}}, \overline{\mathbb{Q}}_{\ell}\right)$ est en degrés au moins $d-1$ (ce corollaire utilise une dualité de Poincaré). On obtient le résultat en prenant la limite inductive sur $m$.

6.2. Calcul de la partie supercuspidale de la cohomologie. - Il s'agit du résultat suivant : la partie supercuspidale est concentrée en degré médian 
$d-1$ et les composantes $\pi$-isotypiques, pour $\pi$ supercuspidale et définies par $\mathcal{U}_{c}^{d-1}(\pi)=\operatorname{Hom}_{\mathrm{GL}_{d}(K)}\left(\pi, \mathcal{U}^{d-1}\right)$ sont données par

$$
\begin{aligned}
& \mathcal{U}_{c, \mathcal{D} r}^{d-1}(\pi)=\mathrm{JL}(\pi) \otimes \mathrm{L}\left(\pi \otimes||^{\frac{1-d}{2}}\right), \\
& \mathcal{U}_{c, \mathcal{L} \mathcal{T}}^{d-1}(\pi)=\mathrm{JL}\left(\pi^{\vee}\right) \otimes \mathrm{L}\left(\pi^{\vee} \otimes||^{\frac{1-d}{2}}\right) .
\end{aligned}
$$

Le tableau suivant résume les diverses contributions :

\begin{tabular}{||l||c|c||}
\hline & côté Drinfeld & côté Lubin-Tate \\
\hline \hline corps $p$-adiques & $\begin{array}{c}\text { Harris }([41] ; 1997) \\
\text { complété par Harris-Taylor }([46]) \\
\text { (voir également [45]) }\end{array}$ & $\begin{array}{c}\text { Harris-Taylor } \\
([46] ; 1998)\end{array}$ \\
\hline $\begin{array}{l}\text { cas d'égale } \\
\text { caractéristique } p\end{array}$ & $\begin{array}{c}\text { Hausberger } \\
([47] ; 2000)\end{array}$ & $\begin{array}{c}\text { Boyer } \\
([14] ; 1998)\end{array}$ \\
\hline
\end{tabular}

Remarque 8. - 1. Comme on peut s'en douter, les démonstrations de ces "conjectures de Deligne-Drinfeld-Carayol" sont intimement liés à celles de la correspondance de Langlands locale.

2. Toutes les preuves procèdent par voie globale (i.e. utilisent un résultat d'uniformisation). Des résultats purement locaux existent mais ne donnent que des résultats partiels, ne permettant pas de calculer la partie galoisienne ([33] [66]).

Remarque 9. - Dans le cas Lubin-Tate, les auteurs calculent en fait, ce qui est plus naturel dans leur contexte, la partie $\rho$-isotypique du dual de $\mathcal{U}_{c, \mathcal{L}}^{d-1}$, pour $\rho$ une représentation de $D^{\times}$, car les cycles evanescents calculent la cohomologie sans support. En d'autres termes, on a côté Lubin-Tate :

$$
\mathcal{U}_{\mathcal{L} \mathcal{T}}^{d-1}\left(\operatorname{JL}\left(\pi^{\vee}\right)\right):=\mathrm{H}^{d-1}\left(\mathcal{M}_{\mathcal{L} \mathcal{T}}^{d} \hat{\otimes} \widehat{\bar{K}}, \overline{\mathbb{Q}}_{\ell}\right)\left(\operatorname{JL}\left(\pi^{\vee}\right)\right) \simeq \pi \otimes \mathrm{L}\left(\pi^{\vee} \otimes||^{\frac{1-d}{2}}\right) .
$$

Suivant Carayol ([17]), nous conservons la symétrie des énoncés.

6.2.1. Schéma de la preuve côté Drinfeld. - On rappelle que $F_{o}$ joue le rôle du corps local $K$ et se donne une représentation supercuspidale $\pi$ de $\mathrm{GL}_{d}\left(F_{o}\right)$. On commence par intégrer la représentation locale $\pi$ au sein d'une représentation globale $\tilde{\Pi}$ qui sera une représentation automorphe de $\mathrm{GL}_{d}(\mathbb{A})$ telle que $\tilde{\Pi}_{o}=$ $\pi$ et $\tilde{\Pi}_{\infty}=S t_{\infty}$ (le type à l'infini doit être de celui qui apparaît dans la cohomologie de la variété globale $\mathcal{E} \ell \ell$, d'où ce choix).

En utilisant des résultats de Henniart (que l'on démontre à l'aide d'une formule des traces), on transfère $\tilde{\Pi}$ au groupe $D^{\times}$(resp. $\left.\bar{D}^{\times}\right)$, en une représentation automorphe $\Pi$ de $D^{\times}(\mathbb{A})\left(\operatorname{resp} . \bar{\Pi}\right.$ de $\left.\bar{D}^{\times}(\mathbb{A})\right)$ telle que $\Pi_{o}=\operatorname{JL}(\pi)$ et $\Pi_{\infty}=S t_{\infty}$ (resp. $\bar{\Pi}_{o}=\pi$ et $\bar{\Pi}_{\infty}=1_{\infty}$ ). Ce sont deux instances d'une correspondance 
de Jaquet-Langlands globale (qui n'était pas disponible en toute généralité à l'époque, avant les travaux récents de Badulescu). Le principe est que les composantes locales ne diffèrent qu'en les places ramifiées où elles se correspondent via la correspondance de Jaquet-Langlands locale. On a donc $\Pi^{\infty, o} \simeq \bar{\Pi}^{\infty, o}$. De plus, on peut supposer (d'après Henniart) que $\Pi$ et $\bar{\Pi}$ sont de multiplicité un forte.

La suite spectrale de Hochschild-Serre

$$
\mathrm{E}_{2}^{i, j}=\operatorname{Ext}_{\mathrm{GL}_{d}\left(F_{o}\right)-\text { lisse }}^{i}\left(\mathcal{U}_{c, \mathcal{D} r}^{2(d-1)-j, \dagger}(d-1), \mathcal{A} \bar{D}\right) \Longrightarrow H_{o}^{i+j}
$$

est $D^{\times}\left(\mathbb{A}^{\infty}\right) \times W_{F_{o}}$-équivariante (on rappelle que l'action de $\mathrm{GL}_{d}\left(F_{o}\right) \operatorname{sur} \mathcal{U}_{c, \mathcal{D} r}{ }^{\dagger}$ est celle de Drinfeld composée avec $\left.g \mapsto{ }^{t} g^{-1}\right)$. De plus, les $\mathrm{E}_{2}^{i, j}$ sont admissibles en tant que représentations de $D^{\times}\left(\mathbb{A}^{\infty}\right) \times W_{F_{o}}$ (c'est une conséquence du fait déjà cité que les groupes de cohomologie à support des revêtements de Drinfeld sont de type fini).

Prendre la composante $\Pi^{\infty, o}$-isotypique dans la suite spectrale a alors un sens et l'on utilise le fait suivant : $\mathrm{E}_{2}^{i, j}\left(\Pi^{\infty, o}\right)=\operatorname{Ext}_{\mathrm{GL}_{d}\left(F_{o}\right)-\text { lisse }}^{i}\left(\mathcal{U}_{c, \mathcal{D} r}^{2(d-1)-j}(d-1), \pi^{\vee}\right)=$ 0 car $\pi$ est supercuspidale (on a également utilisé la multiplicité forte de $\bar{\Pi}$ ), d'où

$$
(1):=\mathrm{E}_{2}^{0, j}\left(\Pi^{\infty, o}\right) \simeq H_{o}^{j}\left(\Pi^{\infty, o}\right)=:(2) .
$$

Or

$(1)=\operatorname{Hom}_{\mathrm{GL}_{d}\left(F_{o}\right)}\left(\mathcal{U}_{c, \mathcal{D} r}^{2(d-1)-j}(d-1), \pi^{\vee}\right)=\operatorname{Hom}_{\mathrm{GL}_{d}\left(F_{o}\right)}\left(\pi^{\vee}, \mathcal{U}_{c, \mathcal{D} r}^{2(d-1)-j}(d-1)\right)^{*}$

et

$$
(2)= \begin{cases}0 & \text { si } j \neq d-1 \\ \mathrm{JL}(\pi) \otimes \mathrm{L}(\pi)\left(\frac{1-d}{2}\right) & \text { si } j=d-1\end{cases}
$$

d'après Laumon-Rapoport-Stuhler. Cela démontre la conjecture de DrinfeldCarayol.

6.2.2. Le côté Lubin-Tate. - La clef réside dans le fait suivant : les strates non supercuspidales $(h<d)$ sont "géométriquement induites", au sens où il existe un sous-schéma fermé $\mathcal{E} \ell \ell_{I, s_{o}, 1}^{=h}$ de $\mathcal{E} \ell \ell_{I, s_{o}}^{=h}$ stable sous un parabolique $P_{h}\left(F_{o}\right)$ de $\mathrm{GL}_{d}\left(F_{o}\right)$ et tel que $\mathcal{E} \ell \ell_{I, s_{o}}^{=h}=\mathcal{E} \ell \ell_{I, s_{o}, 1}^{=h} \times_{P_{h}\left(\mathcal{O}_{o} / \varpi_{o}^{n}\right)} \mathrm{GL}_{d}\left(\mathcal{O}_{o} / \varpi_{o}^{n}\right)$ où $n=\operatorname{mult}_{o}(I)$. En terme de représentations, il en résulte que la partie supercuspidale de la cohomologie se calcule aux points supersinguliers : notant $\mathcal{E} \ell \ell_{I, \bar{s}_{o}}=\mathcal{E} \ell \ell_{I, s_{o}} \otimes$ $\overline{k(o)}$, la partie supercuspidale de la suite spectrale de stratification

$$
\mathrm{E}_{1}^{p, q}=\mathrm{H}_{c}^{p+q}\left(\mathcal{E} \ell \ell_{I, \bar{s}_{o}}^{=d-p}, \mathrm{R}^{j} \Psi_{\eta_{o}} \overline{\mathbb{Q}}_{\ell}\right) \Longrightarrow \mathrm{H}_{c}^{p+q}\left(\mathcal{E} \ell \ell_{I, \bar{s}_{o}}, \mathrm{R}^{j} \Psi_{\eta_{o}} \overline{\mathbb{Q}}_{\ell}\right)
$$


dégénère, et fournit des isomorphismes

$$
\mathrm{H}_{c}^{q}\left(\mathcal{E} \ell \ell_{I, \bar{s}_{o}}^{=d}, \mathrm{R}^{j} \Psi_{\eta_{o}} \overline{\mathbb{Q}}_{\ell}\right)_{\text {supercusp. }} \simeq \mathrm{H}_{c}^{q}\left(\mathcal{E} \ell \ell_{I, \bar{s}_{o}}^{=}, \mathrm{R}^{j} \Psi_{\eta_{o}} \overline{\mathbb{Q}}_{\ell}\right)_{\text {supercusp }}
$$

Soit donc $\pi$ une représentation supercuspidale de $\mathrm{GL}_{d}\left(F_{o}\right)$. Comme précédemment, on choisit une représentation automorphe $\tilde{\Pi}$ de $\mathrm{GL}_{d}(\mathbb{A})$ telle que $\tilde{\Pi}_{o}=\pi$ et $\tilde{\Pi}_{\infty}=S t_{\infty}$. Puis on transfère aux groupes $D^{\times}$et $\bar{D}^{\times}$, les représentations automophes $\Pi$ et $\bar{\Pi}$ obtenues étant de multiplicité un forte et telles que $\Pi_{o}=\pi$, $\Pi_{\infty}=S t_{\infty}$ et $\bar{\Pi}_{o}=\mathrm{JL}(\pi), \bar{\Pi}_{\infty}=1_{\infty}$. (On rappelle que l'algèbre à division d'invariant $1 / d$ est $\bar{D}_{o}$.) D'après la discussion précédente, la composante $\Pi^{\infty, o} \simeq \bar{\Pi}^{\infty, o}$-isoypique est telle que

$$
\mathrm{H}_{c}^{q}\left(\mathcal{E} \ell \ell_{I, \bar{s}_{o}}^{=}, \mathrm{R}^{j} \Psi_{\eta_{o}} \overline{\mathbb{Q}}_{\ell}\right)\left(\Pi^{\infty, o}\right) \simeq \mathrm{H}_{c}^{q}\left(\mathcal{E} \ell \ell_{I, \bar{s}_{o}}, \mathrm{R}^{j} \Psi_{\eta_{o}} \overline{\mathbb{Q}}_{\ell}\right)\left(\Pi^{\infty, o}\right) .
$$

En particulier, ces groupes de cohomologie sont nuls sauf pour $q=0$ car $\mathcal{E} \ell \ell_{I, \bar{s}_{o}}=\frac{d}{}$

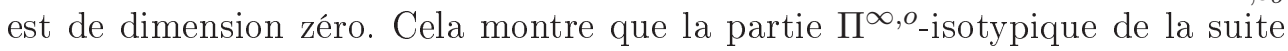
spectrale des cycles évanescents dégénère, d'où un isomorphisme

$$
(1):=\mathrm{E}_{2}^{0, j}\left(\Pi^{\infty, o}\right) \simeq H_{o}^{j}\left(\Pi^{\infty, o}\right)=:(2) .
$$

Or

$$
(1)=\operatorname{Hom}_{\bar{D}_{o}^{\times}}\left(\check{\mathcal{A}}_{\bar{D}}^{\infty}, \widetilde{\mathcal{U}_{\mathcal{L} \mathcal{T}}^{j}}\right)\left(\Pi^{\infty, o}\right)=\operatorname{Hom}_{\bar{D}_{o}^{\times}}\left(\operatorname{JL}\left(\pi^{\vee}\right), \widetilde{\mathcal{U}_{\mathcal{L} \mathcal{T}}^{j}}\right)
$$

d'après la proposition 5.2 et

$$
(2)= \begin{cases}0 & \text { si } j \neq d-1 \\ \pi \otimes \mathrm{L}(\pi)\left(\frac{1-d}{2}\right) & \text { si } j=d-1\end{cases}
$$

d'après Laumon-Rapoport-Stuhler. On obtient le résultat annoncé, sous réserve que la modification de l'action se traduise bien par une contragrédiente pour l'action de $\mathrm{GL}_{d}\left(F_{o}\right) \times \bar{D}_{o}^{\times}$. La conjecture de Deligne-Carayol est démontrée.

Remarque 10. - Par rapport à [14], Boyer tient compte dans [13] de cette modification de l'action.

6.3. Preuve du théorème 8 . - Avant de détailler un peu les travaux récents de Boyer, expliquons comment le théorème 8 se déduit du résultat local principal de [13], [16] et [15], à savoir le :

Théorème 9 (Boyer, cf. [15] 2.3.5 ; voir également [13] IV.4.0.22)

Pour tout diviseur $g$ de $d=s g$ et toute représentation irréductible cuspidale $\pi$ de $\mathrm{GL}_{g}(K)$, on a

$$
\mathcal{U}_{\mathcal{L} \mathcal{T}}^{d-1-i}\left(\mathrm{JL}\left(S t_{s}\left(\pi^{\vee}\right)\right)\right) \simeq \begin{cases}\mathrm{L}\left(\pi^{\vee}\right)\left(-\frac{d+s-2(i+1)}{2}\right) \otimes[\overleftarrow{s-i-1}, \vec{i}]_{\pi} & 0 \leq i<s \\ 0 & i<0\end{cases}
$$


où $[\overleftarrow{s-i-1}, \vec{i}]_{\pi}$ est l'unique sous-espace irréductible de l'induite $S t_{s-i}(\pi) \overrightarrow{\times} \operatorname{Speh}_{i}(\pi)$.

Comme la catégorie des représentations lisses de $D^{\times}$est essentiellement semisimple, on peut écrire

$$
\mathcal{U}_{c, \mathcal{L} T, \chi}^{i}=\bigoplus_{\rho, \chi_{\rho}=\chi} \mathcal{U}_{c, \mathcal{L}}^{i} \mathcal{T}, \chi(\rho) \otimes \rho .
$$

En utilisant la dualité de Poincaré, on obtient

$$
\begin{aligned}
\mathcal{U}_{c, \mathcal{L T}}^{i}(\rho) & =\operatorname{Hom}_{D^{\times}}\left(\rho, \mathcal{U}_{c, \mathcal{L T}}^{i}\right) \simeq \operatorname{Hom}_{D^{\times}}\left(\rho,\left(\mathcal{U}_{\mathcal{L} \mathcal{T}}^{2(d-1)-i}\right)^{*}(1-d)\right) \\
& \simeq \operatorname{Hom}_{D^{\times}}\left(\mathcal{U}_{\mathcal{L} \mathcal{T}}^{2(d-1)-i}, \rho^{\vee}\right)(1-d) \\
& \simeq \operatorname{Hom}_{D^{\times}}\left(\rho^{\vee}, \mathcal{U}_{\mathcal{L T}}^{2(d-1)-i}\right)^{*}(1-d)
\end{aligned}
$$

En résumé, $\left.\mathcal{U}_{c, \mathcal{L} \mathcal{T}}^{d-1+i}(\rho) \simeq\left(\mathcal{U}_{\mathcal{L} \mathcal{T}}^{d-1-i}\right)\left(\rho^{\vee}\right)\right)^{*}(1-d)$ (isomorphisme $\mathrm{GL}_{d}(K) \times W_{K^{-}}$ équivariant). Cela permet de retranscrire le résultat de Boyer en terme de cohomologie avec support, sachant que la duale de $[\overleftarrow{s-i-1}, \vec{i}]_{\pi}$ est $[\vec{i}, \overleftarrow{s-i-1}]_{\pi^{\vee}}$. Or $[\vec{i}, \overleftarrow{s-i-1}]_{\pi}$ est l'unique sous-module irréductible de $\operatorname{Speh}_{i}(\pi) \overrightarrow{\times} S t_{s-i}(\pi)=\operatorname{Speh}_{i}(\pi)||^{\frac{i-s}{2}} \times S t_{s-i}(\pi)||^{\frac{i}{2}}$ que l'on a noté $S_{i, s}(\pi)$ dans l'énoncé du théorème 8 .

\section{Calcul de la cohomologie non supercuspidale}

Nous poursuivons avec les mêmes notations que la preuve du théorème de Deligne-Carayol, toujours en égale caractéristique. Le but de ce paragraphe est d'exposer les grandes lignes de la preuve du théorème 9 donnée par Boyer dans [13].

La première étape consiste à transposer les résultats de Harris et Taylor ([46]) au cas des corps de fonctions, jusqu'au calcul de la représentation virtuelle locale dont la composante $\mathrm{JL}\left(S t_{s}(\pi)\right)$-isotypique s'exprimera :

(1) $\sum_{i=0}^{d-1}(-1)^{i}\left[\widetilde{\mathcal{U}_{\mathcal{L} \mathcal{T}, d}^{d-1-i}}\left(S t_{s}(\pi)\right)\right]=\sum_{i=0}^{s-1}(-1)^{i}\left[S_{i, s}(\pi) \otimes \mathrm{L}(\pi)\left(-\frac{d+s-2(i+1)}{2}\right)\right]$

dans le groupe de Grothendieck. Pour cela, on développe la géométrie afin de "déméler" les strates : c'est le rôle des variétés d'Igusa de première et seconde espèce qui rigidifient respectivement la partie étale et connexe du $\mathcal{O}_{o}$-module $\varpi_{o^{-}}$divisible $G r_{o}(\mathcal{E})$ associé au $\mathcal{D}$-faisceau elliptique $\mathcal{E}$.

On rappelle que la strate $\mathcal{E} \ell \ell_{I, s_{o}}^{=h}$ est géométriquement induite $: \mathcal{E} \ell \ell_{I, s_{o}}^{=h}=$ $\mathcal{E} \ell \ell_{I, s_{o}, 1}^{=h} \times{ }_{P_{h}\left(\mathcal{O}_{o} / \varpi_{o}^{n}\right)} \mathrm{GL}_{d}\left(\mathcal{O}_{o} / \varpi_{o}^{n}\right)$ où $P_{h}\left(F_{o}\right)$ désigne un parabolique de $\mathrm{GL}_{d}\left(F_{o}\right)$ comprenant deux blocs diagonaux de tailles $h$ et $d-h$. Notant 
$n=\operatorname{mult}_{o}(I)$, cette description provient de la structure de niveau de Drinfeld $\eta:\left(\varpi_{o}^{-n} \mathcal{O}_{o} / \mathcal{O}_{o}\right)^{d} \rightarrow G r_{o}\left[\varpi_{o}^{n}\right]$ : comme $G r_{o}\left[\varpi_{o}^{n}\right]$ est isomorphe à $\left(\varpi_{o}^{-n} \mathcal{O}_{o} / \mathcal{O}_{o}\right)^{d-h}$, le noyau de $\eta$ est un facteur direct $a$ de rang $h$ dans $\left(\varpi_{o}^{-n} \mathcal{O}_{o} / \mathcal{O}_{o}\right)^{d}$. On obtient des sous-schémas fermés $\mathcal{E} \ell \ell_{I, s_{o}, a}^{=h}$ et $P_{h}$ opère sur $\mathcal{E} \ell \ell_{I, s_{o}, 1}^{=h}$.

Les variétés d'Igusa de première espèce classifient les structures de niveau partielles $\eta^{e ́ t}:\left(\varpi_{o}^{-n} \mathcal{O}_{o} / \mathcal{O}_{o}\right)^{d-h} \stackrel{\sim}{\rightarrow} G r_{o}^{e ́ t}\left[\varpi_{o}^{n}\right]$. Cela définit un revêtement étale galoisien $\mathcal{I}_{I^{o}, n}^{=h} \rightarrow \mathcal{E} \ell \ell_{I, s_{o}}^{=h}$ de groupe $\mathrm{GL}_{d-h}\left(\mathcal{O}_{o} / \varpi_{o}^{n} \mathcal{O}_{o}\right)$. Or on peut démontrer que l'extension $1 \rightarrow G r_{o}^{e ́ t} \rightarrow G r_{o} \rightarrow G r_{o}^{c} \rightarrow 1$ est scindée modulo $\varpi_{o}^{n}$ après torsion par la puissance $\operatorname{Frob}_{o}^{d h}$ du Frobenius. Cela permet d'envoyer $\mathcal{I}_{I^{o}, n}^{=h}$ dans $\mathcal{E} \ell \ell_{I, s_{o}, a}^{=h}$, où la structure de niveau $\eta$ se décompose en $\eta^{e ́ t}:\left(\varpi_{o}^{-n} \mathcal{O}_{o} / \mathcal{O}_{o}\right)^{d} / a \rightarrow$ $G r_{o}^{e ́ t}\left[\varpi_{o}^{n}\right]$ et $\eta^{c}: a \rightarrow G r_{o}^{c}\left[\varpi_{o}^{n}\right]$, de sorte que le diagramme suivant est commutatif

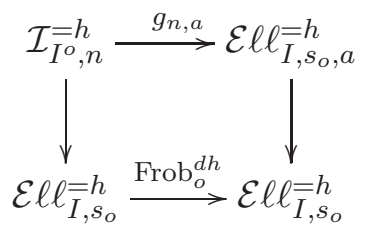

et $g_{n, a}$ est une bijection sur le schéma réduit $\mathcal{E} \ell_{I, s_{o}, a, r e d}^{=h}$ au niveau des points géométriques. Les variétés $\mathcal{I}_{I^{o}, n}^{=h}$ constituent un système projectif avec des morphismes de transition (définis de sorte que les diagrammes précédents commutent) $\mathcal{I}_{I^{o}, n}^{=h} \stackrel{\operatorname{Frob}_{o}^{\left(n-n^{\prime}\right) h}}{\longrightarrow} \mathcal{I}_{I^{o}, n^{\prime}}^{=h}$, et sont munies d'une action par correspondances de $\mathrm{GL}_{d-h}\left(F_{o}\right)$.

On introduit ensuite des variantes formelles : en prenant le complété formel le long de la strate $\mathcal{E} \ell \ell_{I, s_{o}}$, on obtient des extensions formelles étales $\widehat{\mathcal{I}}_{I^{o},=h, n} \rightarrow{\widehat{\mathcal{E} \ell l_{I^{o}},=h}}$, de fibre spéciale $\mathcal{I}_{I^{o}, n}^{=h} \rightarrow \mathcal{E} \ell \ell_{I, s_{o}}^{=h}$, classifiant les structures de niveau partielles $\eta^{e ́ t}$ au-dessus de ${\widehat{\mathcal{E} \ell \ell_{I^{o}}}}_{=h}$. On définit alors le schéma formel $\widehat{\mathcal{I}}_{I^{o},=h, n}(t)$ classifiant les structures de niveau (de Drinfeld) partielles $\eta^{c}$ : $\left(\varpi_{o}^{-n} \mathcal{O}_{o} / \mathcal{O}_{o}\right)^{t} \rightarrow G r_{o}^{c}\left[\varpi_{o}^{n}\right]$. Il s'agit d'un revêtement très ramifié de $\widehat{\mathcal{I}}_{I^{o},=h, n}$. Puis on étend le morphisme $g_{n, a}$ en un morphisme $\hat{g}_{n, a}: \widehat{\mathcal{I}}_{I^{o},=h, n}(n) \rightarrow{\widehat{\mathcal{E} \ell \ell_{I,}=h, a}}$ faisant commuter le diagramme précédent (étendu aux complétés). Pour des raisons de dimension, $\hat{g}_{n, a}$ est un isomorphisme. On définit ensuite l'action par correspondances de $\mathrm{GL}_{d-h}\left(F_{o}\right) \times \mathrm{GL}_{h}\left(F_{o}\right)$ sur la tour $\left(\widehat{\mathcal{I}}_{I^{o},=h, n}\right)_{n}$, de façon compatible à l'action sur $\mathcal{I}_{I^{o}, n}^{=h}$ et aux morphismes $\hat{g}_{n, a}$.

En un point géométrique $\bar{z}_{o}$ de $\mathcal{I}_{I^{o}, n}^{=h}$, la fibre de $\widehat{\mathcal{I}}_{I^{o},=h, n}(t)$ s'identifie à l'espace des déformations de $\left(G r_{o}\right)_{\bar{z}_{o}}$ muni d'une structure $\eta^{c}$ partielle de niveau 
$t$. Le choix d'un isomorphisme $\left(G r_{o}^{c}\right)_{\bar{z}_{o}} \simeq \Phi_{h}$ induit un isomorphisme entre $\left(\widehat{\mathcal{I}}_{I^{o},=h, n}(t)\right)_{\bar{z}_{o}}$ et $\operatorname{Spf} \operatorname{Def}_{t}^{h} \simeq \widehat{\mathcal{M}}_{\mathcal{L} \mathcal{T}, t}^{h,[0]}$. Cependant, le groupe $G r_{o}^{c}$ n'est pas constant sur le système projectif des $\mathcal{I}_{I^{o}, n}^{=h}$, si bien qu'un tel isomorphisme n'existe pas globalement. C'est pourquoi l'on introduit les variétés d'Igusa de seconde espèce.

Il s'agit de revêtements étales galoisiens $\mathcal{J}_{I^{o}, n}^{=h}(s) \rightarrow \mathcal{I}_{I^{o}, n}^{=h} \otimes \overline{k(o)}$ de groupe $\left(\mathcal{O}_{D_{h}} / \varpi^{s} \mathcal{O}_{D_{h}}\right)^{\times}$obtenus par des rigidifications $G r_{o}^{c}\left[\varpi^{s}\right] \simeq \Phi_{h}\left[\varpi^{s}\right]$. Par le système projectif $\mathcal{J}_{I^{o}, n}^{=h}(\infty)$ de groupe $\mathcal{O}_{D_{h}}^{\times}$, on associe à toute représentation admissible $\tau$ de $\mathcal{O}_{D_{h}}^{\times}$un système local $\mathcal{F}(\tau)$, c'est-à-dire un $\overline{\mathbb{Q}}_{\ell}$-faisceau lisse obtenu comme quotient du faisceau constant de fibre $\tau$ par l'action de $\mathcal{O}_{D_{h}}^{\times}$. Par ailleurs, la (double) tour $\left(\mathcal{J}_{I^{o}, n}^{=h}(s)\right)_{n, s}$ est munie d'une action par correspondances de $\left(\mathrm{GL}_{h}\left(F_{o}\right) \times D_{h}^{\times} \times W_{F_{o}}\right)^{1} \times \mathrm{GL}_{d-h}\left(F_{o}\right)$.

Avec les notations du paragraphe 5.2, on considère les cycles évanescents $\mathrm{R} \Psi_{\eta_{o}} \overline{\mathbb{Q}}_{\ell}$ associés à $\mathcal{E} \ell \ell_{I}$ (où $n=$ mult $_{o}(I)$ ) ainsi que les cycles évanescents formels $\mathrm{R} \Psi^{\text {form }} \overline{\mathbb{Q}}_{\ell}$ associés à $\operatorname{Def}_{n}^{h}$. On pose $\Psi_{h, n}^{i}=\mathrm{H}^{0}\left(\mathrm{R}^{i} \Psi^{\text {form }} \overline{\mathbb{Q}}_{\ell}\right)$, sur lequel agit $\left(\mathrm{GL}_{h}\left(F_{o}\right) \times D_{h}^{\times} \times W_{F_{o}}\right)^{1}$, et $\mathcal{U}_{h, n}^{i}=\operatorname{Ind}_{\left(\mathrm{GL}_{h}\left(F_{o}\right) \times D_{h}^{\times} \times W_{F_{o}}\right)^{1}}^{\mathrm{GL}_{h}\left(F_{o}\right) \times D_{h, n}^{\times} \times W_{F_{o}}} \Psi^{i}$, de sorte que la représentation locale fondamentale est $\mathcal{U}_{\mathcal{L} T, h}^{i}=\underline{h}_{h} \mathcal{U}_{h, n}^{i}$.

On s'intéresse à $\mathcal{F}\left(\Psi_{h, n}^{i}\right)$. D'après ce qui précède, la restriction à $\mathcal{E} \ell \ell_{I, \bar{s}_{o}, 1}^{h}$ de $\mathrm{R}^{i} \Psi_{\eta_{o}} \overline{\mathbb{Q}}_{\ell}$ coïncide avec les faisceaux évanescents associés à $\widehat{\mathcal{I}}_{I^{o},=h, n}(n)$ (via $\left.\hat{g}_{n, 1}\right)$, que nous noterons $\Psi_{h, n}^{\prime i}$. En un point géométrique $\bar{z}_{o}$ de $\mathcal{I}_{I^{o}, n}^{h} \otimes \overline{k(o)}$ on a donc un isomorphisme de fibres

$$
\left(\mathrm{R}^{i} \Psi_{\eta_{o}} \overline{\mathbb{Q}}_{\ell}\right)_{\bar{z}_{o}} \simeq\left(\Psi_{h, n}^{\prime i}\right)_{\bar{z}_{o}} \simeq\left(\mathcal{F}\left(\Psi_{h, n}^{i}\right)\right)_{\bar{z}_{o}}
$$

En effet, un point de $\left(\Psi_{h, n}^{\prime i}\right)_{\bar{z}_{o}}$ étant donné, il correspond à tout oùn $\bar{z}_{o}(\infty)$ de $\bar{z}_{o}$ dans $\mathcal{J}_{I^{o}, n}^{h}(\infty)$ la donnée d'un isomorphisme $G r_{o}^{c} \simeq \Phi_{h}$, donc d'un isomorphisme $\left.\left(\Psi_{h, n}^{\prime i}\right)_{\bar{z}_{o}} \simeq\left(\Psi_{h, n}^{i}\right)\right)_{\bar{z}_{o}}\left(\operatorname{via}\left(\widehat{\mathcal{I}}_{I^{o},=h, n}(n)\right)_{\bar{z}_{o}} \simeq \operatorname{Def}_{n}^{h}\right)$, d'où finalement un point de $\left(\Psi_{h, n}^{i}\right)_{\bar{z}_{o}}$, et ceci de façon compatible à l'action de $\mathcal{O}_{D_{h}}^{\times}$. D'après un résultat de Bekovich (voir l'appendice de [46]), on a en fait un isomorphisme de faisceaux $\left.\mathrm{R}^{i} \Psi_{\eta_{o}} \overline{\mathbb{Q}}_{\ell}\right|_{\mathcal{E} \ell=h} \simeq \mathcal{F}\left(\Psi_{h, n}^{i}\right)$.

Décomposant l'action de $\mathcal{O}_{D_{h}}^{\times}$sur $\Psi_{h, n}^{i}$ en composantes isotypiques, on obtient une décomposition du faisceau $\mathcal{F}\left(\Psi_{h, n}^{i}\right)=\bigoplus_{\tau} \mathcal{F}(\tau) \otimes \Psi_{h, n}^{i}(\tau)$, d'où finalement des isomorphismes

(2) $\quad{\underset{\lim }{I \not \supset \infty}}_{H_{c}^{j}}\left(\mathcal{E} \ell \ell_{I, \bar{s}_{o}, 1}^{\bar{h}}, \mathrm{R}^{i} \Psi \overline{\mathbb{Q}}_{\ell}\right)^{h} \simeq \bigoplus_{\tau}\left(\underset{I \not \supset \infty}{\lim _{I \not \infty}} \mathrm{H}_{c}^{j}\left(\mathcal{E} \ell \ell_{\bar{I}, \bar{s}_{o}, 1}^{\bar{h}}, \mathcal{F}_{\tau}\right) \otimes \widetilde{\mathcal{U}_{\mathcal{L T}, h}^{i}}(\tau)\right)^{\frac{h}{e_{\tau}}}$ 
où la somme porte sur les classes d'équivalence inertielle de représentations admissibles irréductibles de $D_{h}^{\times}$. On a utilisé la réciprocité de Frobenius $\operatorname{Hom}_{D_{h}^{\times}}\left(\tau, \mathcal{U}_{h, n}^{i}\right)=\operatorname{Hom}_{\mathcal{O}_{D_{h}}^{\times}}\left(\left.\tau\right|_{\mathcal{O}_{D_{h}}^{\times}}, \Psi_{h, n}^{i}\right)$ pour faire apparaêtre $\mathcal{U}_{\mathcal{L} T, h}^{i}$. La formulation un peu compliquée provient du fait que la restriction $\left.\tau\right|_{\mathcal{O}_{D_{h}}^{\times}}$se décompose a priori en un nombre fini $e_{\tau}$ (divisant $h$ ) de composantes irréductibles $\rho_{i}$ et $\rho_{j}$ coïncide avec $\rho_{i}$ sur $\mathcal{O}_{D_{h}}^{\times}$si et seulement si $\rho_{j}=\rho_{i} \circ \xi \circ v \circ \mathrm{Nrd}$ pour un caractère $\xi: \mathbb{Z} \rightarrow \overline{\mathbb{Q}}_{\ell}^{\times}$(équivalence inertielle). Bien que nous n'ayons pas suivi les actions, ces isomorphismes sont équivariants pour l'action de $D^{\times}\left(\mathbb{A}^{\infty, o}\right) \times \mathrm{GL}_{h}\left(F_{o}\right) \times \mathrm{GL}_{d-h}\left(F_{o}\right) \times W_{F_{o}}$. Précisons que $\lim _{\longrightarrow}{ }_{\not \ngtr \infty} \mathrm{H}_{c}^{j}\left(\mathcal{E} \ell \ell_{I, \bar{s}_{o}, 1}^{=h}, \mathcal{F}(\tau)\right)$ est vue comme une représentation de $D^{\times}\left(\mathbb{A}^{\infty, o}\right) \times \mathrm{GL}_{d-h}\left(F_{o}\right) \times \mathbb{Z}$ via le quotient $\left(\mathrm{GL}_{h}\left(F_{o}\right) \times D_{h}^{\times} \times W_{F_{o}}\right)^{0} / \mathcal{O}_{D_{h}}^{\times}$ isomorphe à $\mathbb{Z}$. Un élément $\left(g^{\infty, o}, g_{o}^{c}, g_{o}^{e ́ t}, \sigma_{o}\right)$ appartenant au grand groupe cidessus agit alors via $\left(g^{\infty, o}, g_{o}^{e ́ t},-v\left(\operatorname{det} g_{o}^{c}\right)-v\left(c l\left(\sigma_{o}\right)\right)\right) \otimes\left(g_{o}^{c}, \sigma_{o}\right)$ sur le produit tensoriel du second membre de (2). Pour être exact, il faut également modifier l'action de $\mathrm{GL}_{d}\left(F_{o}\right)$ sur la représentation locale fondamentale, ce qui est matérialisé comme d'habitude par la notation $\widetilde{\mathcal{U}_{\mathcal{L T}, h}^{i}}$. C'est pour cela également qu'apparaît le groupe $\left(\mathrm{GL}_{h}\left(F_{o}\right) \times D_{h}^{\times} \times W_{F_{o}}\right)^{0}$ et non $\left(\mathrm{GL}_{h}\left(F_{o}\right) \times D_{h}^{\times} \times W_{F_{o}}\right)^{1}$. On considère maintenant la représentation virtuelle globale

$$
\mathrm{H}_{o}^{*}=\sum_{i=0}^{2 d-2}(-1)^{d-1-i} \underset{I \not \supset \infty}{\lim _{I}} \mathrm{H}^{i}\left(\mathcal{E} \ell \ell_{I} \otimes \bar{F}_{o}, \overline{\mathbb{Q}}_{\ell}\right) .
$$

D'après la suite spectrale des cycles évanescents et celle de stratification, nous avons

$$
\mathrm{H}_{o}^{*}=\sum_{j=0}^{d-1} \sum_{h=1}^{d}(-1)^{j} \underset{I \not \supset \infty}{\lim _{C}} \mathrm{H}_{c}^{*}\left(\mathcal{E} \ell \ell_{I, \bar{s}_{o}}^{=h}, R^{j} \Psi_{\eta_{o}} \overline{\mathbb{Q}}_{\ell}\right) .
$$

Sachant que

$$
\mathrm{H}_{c}^{*}\left(\mathcal{E} \ell \ell_{I, \bar{s}_{o}}^{=h}, \mathrm{R}^{j} \Psi_{\eta_{o}} \overline{\mathbb{Q}}_{\ell}\right)=\operatorname{Ind}_{P_{h}\left(F_{o}\right)}^{\mathrm{GL}_{d}\left(F_{o}\right)} \mathrm{H}_{c}^{*}\left(\mathcal{E} \ell \ell_{I, \bar{s}_{o}, 1}^{=h}, \mathrm{R}^{j} \Psi_{\eta_{o}} \overline{\mathbb{Q}}_{\ell}\right)
$$

(égalité qui commute à la limite sur $I$ ), on peut utiliser l'isomorphisme (2). Soit donc $s$ un diviseur de $d, \pi_{o}$ une représentation supercuspidale de $\operatorname{GL}_{\frac{d}{s}}\left(F_{o}\right)$ et $\Pi$ une représentation automorphe (de multiplicité un forte) de $D^{\times}(\mathbb{A})$ telle que $\Pi_{\infty}=S t_{\infty}$ et $\Pi_{o}=S t_{s}\left(\pi_{o}\right)$. On prend d'autre part $\tau=\operatorname{JL}\left(S t_{s}\left(\pi_{o}\right)\right)$. La composante $\Pi^{\infty, 0}$-isotypique de $\mathrm{H}_{o}^{*}$ a déjà été calculée dans [54] : on trouve $\Pi_{o} \otimes \mathrm{L}\left(\Pi_{o}\right)$. La démarche est classique : on compte par la formule des traces de Lefschetz les points fixes sous l'action d'une correspondance de Hecke tordue 
par une puissance assez grande de Frobenius, puis transfert les intégrales orbitales obtenues afin de reconnaître le côé géométrique de la formule des traces de Selberg.

Boyer adapte alors ces méthodes pour calculer la composante $\Pi^{\infty, o_{-}}$-isotypique

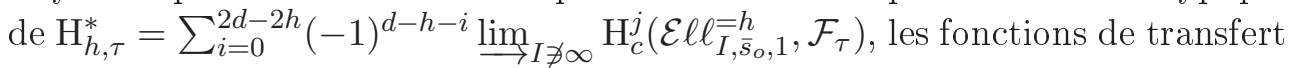
étant fournies par Harris et Taylor. Si $V$ désigne l'espace de la représentation $\tau$ et $s$ un entier suffisamment grand pour que $\tau$ soit trivial sur $1+\varpi_{0}^{s} \mathcal{O}_{D_{h}}$, alors $\mathrm{H}_{c}^{j}\left(\mathcal{E} \ell \ell_{I, \bar{s}_{o}, 1}^{=h}, \mathcal{F}_{\tau}\right) \simeq \mathrm{H}_{c}^{j}\left(\mathcal{J}_{I^{o}, n}^{=h}(s), \overline{\mathbb{Q}}_{\ell}\right) \otimes V^{\left(\mathcal{O}_{D_{h}} / \varpi_{o}^{s} \mathcal{O}_{D_{h}}\right)^{\times}}$. On applique les formules des traces, après avoir obtenu une description adélique de $\mathcal{J}_{I^{o}, n} h(s)(\overline{k(o)})$.

Enfin, on injecte le résultat dans l'égalité (3). Il en résulte une formule de récurrence qui permet de démontrer le résultat annoncé (1), moyennant le calcul d'une induite parabolique, qui fait justement apparaître les $S_{i, s}\left(\pi_{o}\right)$.

Il s'agit maintenant de montrer qu'aucune annulation ne se produit dans l'égalité (1) : c'est là que réside la contribution originale de Boyer, au sens où l'on a essentiellement jusqu'à présent traduit dans le language des variétés de Laumon-Rapoport-Stuhler les résultats de Harris et Taylor.

C'est un travail très délicat, technique et calculatoire. Essayons, autant que faire se peut, d'en mettre en avant la portée conceptuelle et les ingrédients. Le contexte catégoriel est celui des $W_{F_{o}}$-faisceaux pervers de Hecke, dont les objets sont des tours $\left(\mathcal{F}_{I}\right)_{I \in \mathcal{I}}$ de $\overline{\mathbb{Q}}_{\ell}$-faisceaux pervers (voir [3] où ces derniers sont définis comme le coeur de la catégorie triangulée munie de la $t$-structure de perversité(1) $\mathcal{F}_{I}$ munies d'une action de $D^{\times}\left(\mathbb{A}^{\infty}\right) \times W_{F_{o}}$ par les correspondances de Hecke, l'action de l'inertie étant supposée potentiellement unipotente sur chaque $\mathcal{F}_{I}$. C'est une catégorie abélienne, munie des foncteurs usuels $\left(j_{\mathcal{I}, !}\right.$, $R j_{\mathcal{I}, *}, j_{\mathcal{I}, ! *}=\operatorname{Im}\left(j_{\mathcal{I}, !} \rightarrow R j_{\mathcal{I}, *}\right)$ et des versions perverses ${ }^{p} j_{\mathcal{I}}^{*}={ }^{p} j_{\mathcal{I}}^{!}$pour toute immersion ouverte affine $j_{\mathcal{I}}: U_{\mathcal{I}} \hookrightarrow X_{\mathcal{I}}$, etc.) et de la dualité de Verdier. On utilisera le yoga habituel sur les faisceaux pervers : par exemple, pour tout $i$, $h^{i} \mathcal{F}_{I}$ a un support de dimension inférieure ou égale à $-i$. Le foncteur $j_{\text {!* }}$ (extension intermédiaire) est particulièrement important car il permet de définir les constituants simples de $\mathcal{F}_{I}$ : un tel faisceau pervers se dévisse en faisceaux pervers simples $j_{!_{*}} \mathcal{L}_{I}$ associés à des systèmes locaux $\mathcal{L}_{I}$ sur des ouverts $U_{I}$. L'exemple typique qui nous concerne est le complexe $\Psi_{\mathcal{I}}=R \Psi_{\eta_{o}} \overline{\mathbb{Q}}_{\ell}[d-1]\left(\frac{d-1}{2}\right)$ des cycles évanescents globaux sur le schéma de Hecke $X=\mathcal{E} \ell_{\mathcal{I}, \bar{s}_{o}}$ (après décalage et torsion). La stratification par les schémas localement fermés $X^{=h}=$

\footnotetext{
$\overline{{ }^{(1)} \text { Les faisceaux }}$ pervers n'etant ni des faisceaux, ni pervers, la terminologie requiert une explication. [3] p. 10
} 
$\mathcal{E} \ell \ell_{\overline{\mathcal{I}}, \bar{s}_{o}}^{s}$ qui partitionnent $X$ s'exprime comme une suite d'inclusions $X^{\geq d} \subset$ $\ldots \subset X^{\geq 1}=X$ où $X^{\geq h}$ désigne l'adhérence schématique de $X^{=h}$. On note $i^{h}$ et $j^{\geq h}$ les inclusions respectives $X^{\geq h} \hookrightarrow X$ et $X^{=h} \hookrightarrow X^{\geq h}$. Par perversité, l'image de $\Psi_{\mathcal{I}}$ dans le groupe de Grothendieck des faisceaux pervers est déterminée par celle de $\sum_{i}(-1)^{i}\left[\left.R^{i} \Psi_{\mathcal{I}}\right|_{X=h}\right]$ dans le groupe de Grothendieck des faisceaux localement constants. Or l'isomorphisme (2) peut se réécrire comme un isomorphisme $D^{\times}\left(\mathbb{A}^{\infty}\right) \times W_{F_{o}}$-équivariant

$$
\left(\left.\mathrm{R}^{i} \Psi_{\mathcal{I}}\right|_{X=h}\right)^{h} \simeq \bigoplus_{\tau}\left(\mathcal{F}(\tau)_{\mathcal{I}} \otimes \widetilde{\mathcal{U}_{\mathcal{L} T, h}^{i}}(\tau)\right)^{\frac{h}{e_{\tau}}}
$$

où l'on note encore $\mathcal{F}(\tau)$ le système local $\mathcal{F}(\tau) \times_{P_{h}\left(\mathcal{O}_{o} / \varpi_{o}^{n} \mathcal{O}_{o}\right)} \mathrm{GL}_{d}\left(\mathcal{O}_{o} / \varpi_{o}^{n} \mathcal{O}_{o}\right)$ sur $X^{=h}$ induit à partir de $\mathcal{F}(\tau)$ sur $\mathcal{E} \ell \ell_{I, \bar{s}_{o}, 1}^{=h}$ (on a posé $\operatorname{mult}_{o}(I)=n$ ).

Cela conduit à définir les $W_{o}$-faisceaux pervers de Hecke sur $X_{\mathcal{I}}^{=s g}$, que Boyer note $H T\left(\pi_{o}, \Pi_{s}\right)$, associés à une représentation supercuspidale $\pi_{o}$ de $\mathrm{GL}_{g}\left(F_{o}\right)$ et une représentation $\Pi_{s}$ de $\mathrm{GL}_{s g}\left(F_{o}\right)$, pour $1 \leq g \leq d$ et $1 \leq s \leq s_{g}$ où $s_{g}=\left\lfloor\frac{d}{g}\right\rfloor$, par la formule

$$
H T\left(\pi_{o}, \Pi_{s}\right)=\mathcal{F}\left(\operatorname{JL}\left(S t_{s}\left(\pi_{o}\right)\right)\right)\left(\frac{d-s g}{2}\right) \otimes \Pi_{s}[d-s g],
$$

où l'action du Levi $\mathrm{GL}_{s g}\left(F_{o}\right) \times \mathrm{GL}_{d-s g}\left(F_{o}\right)$ (le radical unipotent agit trivialement) est donnée par celle sur $\mathcal{F}\left(\mathrm{JL}\left(S t_{s}\left(\pi_{o}\right)\right)\right)$ et l'action de $\mathrm{GL}_{s g}\left(F_{o}\right)$ sur $\Pi_{s}$, le groupe de Weil agissant via son quotient $\mathbb{Z}$ sur $\mathcal{F}\left(\operatorname{JL}\left(S t_{s}\left(\pi_{o}\right)\right)\right)$ de façon tordue par le caractère $\left(\frac{d-s g}{2}\right)$. En particulier, on prend $\Pi_{s}=S t_{s}\left(\pi_{o}\right)$ et définit les $W_{o}$-faisceaux pervers de Hecke sur $X^{\geq h}$

$$
\mathcal{P}\left(s, \pi_{o}\right)=j_{\mathcal{I}, ! *}^{\geq s g} H T\left(\pi_{o}, S t_{s}\left(\pi_{o}\right) \otimes \mathrm{L}\left(\pi_{o}\right) .\right.
$$

(ce sont bien des faisceaux pervers car $j^{\geq h}$ est une immersion affine).

Pour $\mathcal{F}$ un $W_{o}$-faisceau pervers, Boyer note $\mathcal{F}_{\pi_{o}}$ la partie $\pi_{o}$-isotypique, c'està-dire la composante $\left.\mathrm{L}\left(\pi_{o}\right)\right|_{I_{o}}$-isotypique de $\mathcal{F}$. Avec ces notations et les remarques précédentes, on a l'égalité suivante dans le groupe de Grothendieck

$$
e_{\pi_{o}}\left[\Psi_{\mathcal{I}, \pi_{o}}\right]=\sum_{s=1}^{s_{g}} \sum_{i=0}^{s-1}(-1)^{i}\left[j_{\mathcal{I}, !}^{\geq s g} H T\left(\pi_{o}, S_{i, s}\left(\pi_{o}\right)\right) \otimes \mathrm{L}\left(\pi_{o}\right)\left(-\frac{s-2 i-1}{2}\right)\right]
$$

en vertu de (4) et (1), où $e_{\pi_{o}}$ désigne le cardinal de la classe d'équivalence inertielle de $\pi_{o}$. 
Partant de cette égalité, une partie du travail est de démontrer le résultat plus précis suivant :

$$
e_{\pi_{o}}\left[\Psi_{\mathcal{I}, \pi_{o}}\right]=\sum_{s=1-s_{g}}^{s_{g}-1} \sum_{\substack{|s|<t \leq s_{g} \\ t \equiv s-1}}\left[\mathcal{P}\left(t, \pi_{o}\right)\left(-\frac{s}{2}\right)\right]
$$

Pour cela, on étudie la monodromie $N$, à laquelle est associée la filtration de monodromie (de longueur finie) $\cdots \subset M_{i} V \subset M_{i+1} V \subset \cdots$, où $V$ désigne l'espace de la représentation de $W_{F_{o}}$ considérée, caractérisée par le fait que $N\left(M_{i} V\right) \subset M_{i-2} V$ et que $N^{i}$ induit un isomorphisme $g r_{i} V \simeq g r_{-i} V$ au niveau des gradués. Rappelons également qu'il existe une seconde filtration liée à l'action de $W_{F_{o}}$ (mais cette fois au Frobenius et non à l'inertie), la filtration par les poids que nous noterons $\cdots \subset W_{i} V \subset W_{i+1} V \subset \cdots$, introduite par Deligne et caractérisée par le fait que les gradués $g r_{i}^{W}$ sont de poids $i$, c'est-à-dire que les valeurs propre d'un relèvement du Frobenius géométrique agissant sur $g r_{i}^{W}$ sont des entiers algébriques de norme $q^{i / 2}$. La conjecture de monodromie-poids dit que $M_{i}\left(H^{j}\left(X_{\bar{F}_{o}}, \overline{\mathbb{Q}}_{\ell}\right)=W_{i+j}\left(H^{j}\left(X_{\bar{F}_{o}}, \overline{\mathbb{Q}}_{\ell}\right)\right)\right.$ si $X$ est propre et lisse sur $K$. Elle est connue en égale caractéristique suite aux travaux de Deligne sur les conjectures de Weil. Si maintenant on considère le cas du complexe $\mathrm{R} \Psi \overline{\mathbb{Q}}_{\ell}$ des cycles évanescents, l'action de l'inertie définit également un endomorphisme nilpotent dans la catégorie des faisceaux pervers, d'où un objet filtré dans la catégorie dérivée des faisceaux sur la fibre spéciale. Notant $g r_{k}$ les gradués associés à $\Psi_{\mathcal{I}}$, et $g r_{k, \pi_{o}}$ leur partie $\pi_{o}$-isotypique, on dispose de suites spectrales $D^{\times}\left(\mathbb{A}^{\infty}\right)$-équivariantes

$$
E_{1, g r, g l o b, \pi_{o}}^{i, j}=h^{i+j} g r_{-i, \pi_{o}} \Longrightarrow \mathrm{R}^{i+j+d-1} \Psi_{\pi_{o}} .
$$

Le théorème de monodromie-poids dit dans ce contexte que $g r_{i, \pi_{o}}$ est pur de poids $i$. Il en résulte directement, compte-tenu de (6) et du fait que les $\mathcal{P}\left(t, \pi_{o}\right)$ sont purs de poids zéro, l'égalité

$$
e_{\pi_{o}} g r_{i, \pi_{o}}=\bigoplus_{\substack{|i|<t \leq s_{g} \\ t \equiv i-1 \quad \bmod 2}} \mathcal{P}\left(i, \pi_{o}\right)\left(-\frac{i}{2}\right) .
$$

Si $\bar{z}_{o}$ désigne un point géométrique de la fibre spéciale, la fibre $\bar{z}_{o}^{*} \Psi_{I}$ est munie d'une action de $N$ mais n'est pas a priori un faisceau pervers. Le complexe des cycles évanescents formels $\mathrm{R} \Psi^{\text {form }} \overline{\mathbb{Q}}_{\ell}$ l'est par contre, et Fargues étend dans l'appendice [38] l'isomorphisme $\bar{z}_{0}^{*} \Psi_{I} \simeq \mathrm{R} \Psi^{\text {form }} \overline{\mathbb{Q}}_{\ell}$ à la filtration de monodromie : on a des isomorphismes $\bar{z}_{o}^{*} g r_{k} \simeq g r_{k}^{f o r m}$, ainsi qu'au niveau des termes des suites spectrales associées. Or on a vu que si $\bar{z}_{o}$ est un point géométrique 
de la strate $\mathcal{E} \ell \ell_{I, \bar{s}_{o}}^{=h}$, alors $\mathrm{R} \Psi^{\text {form }} \overline{\mathbb{Q}}_{\ell}$ calcule la cohomologie de la tour $\mathcal{M}_{\mathcal{L} T, h}^{[0]}$. En posant $g r_{k, l o c, h, \pi_{o}}=\operatorname{Ind}_{\left(\mathrm{GL}_{h}\left(F_{o}\right) \times D_{h}^{\times} \times W_{F_{o}}\right)^{1}} \overline{\mathrm{GL}}_{o}\left(F_{o}\right){ }^{\times} r_{k, \pi_{o}}$, on a donc une suite spectrale de monodromie locale

$$
E_{1, g r, l o c, h, \pi_{o}}^{i, j}=h^{i+j} g_{-i, l o c, h, \pi_{o}} \Longrightarrow \mathcal{U}_{\mathcal{L} \mathcal{T}, h, \pi_{o}}^{i+j+d}\left(\frac{d-1}{2}\right) .
$$

On prend $h=d=s g$. Le théorème 8 décrivant la représentation locale fondamentale de Deligne-Carayol résulte alors directement du fait que la suite spectrale (7) dégénère en $E_{2}$, donc également la suite spectrale locale (9), comptetenu du résultat de Berkovich-Fargues énoncé plus haut. Précisément, le calcul de $E_{2, g r, l o c, d, \pi_{o}}^{i, j}$ déduit de celui de $E_{2, g r, g l o b, \pi_{o}}^{i, j}$ montre que les termes non nuls sont de la forme $E_{2, g r, l o c, d, \pi_{o}}^{i-s+1, s-2 i} \simeq S_{i, s}\left(\pi_{o}\right) \otimes \bar{z}_{o}^{*} \mathcal{F}\left(s, \pi_{o}\right) \otimes \mathrm{L}\left(\pi_{o}\right)\left(-\frac{s(g+1)-2(i+1)}{2}\right)$, la représentation $\bar{z}_{o}^{*} \mathcal{F}\left(s, \pi_{o}\right)$ de $D_{d}^{\times}$restant encore à déterminer $\left(\operatorname{car} \mathcal{F}\left(s, \pi_{o}\right)\right.$ provient en fait de la restriction de $\operatorname{JL}\left(S t_{s}\left(\pi_{o}\right)\right)$ à $\mathcal{O}_{D_{d}}^{\times}$, et n'est donc pas connue entièrement a priori), ce que l'on peut faire en travaillant dans le groupe de Grothendieck, en comparant avec (1). Bien entendu, on trouve $\mathrm{JL}\left(S t_{s}\left(\pi_{o}\right)\right)$. Il reste à expliquer comment on démontre (6) à partir de (5) et comment on calcule les termes de la suite spectrale de poids globale à partir de (8). Le principe est encore une récurrence sur $d$ : on suppose donc connu le théorème 8 , ainsi que l'expression des $h^{i} g r_{k, l o c, h, \pi_{o}}$ pour tout $h<d$, c'est-à-dire les germes des $h^{i} g r_{k, \pi_{o}}$ en les points non supersinguliers.

Expliquons, de manière vague, comment Boyer procède. Il commence par montrer que $g r_{k, \pi_{o}}$ diffère du résultat attendu (8), dans le groupe de Grothendieck, d'une somme de faisceaux pervers concentrés en les points supersinguliers. Ces derniers sont des faisceaux pervers ponctuels déterminés complètement par le $H^{0}$. S'en suivent des calculs cohomologiques aboutissant, en corollaire, au calcul de certains groupes $\lim _{\longrightarrow \not \supset \infty} H_{c}^{i}\left(\mathcal{E} \ell \ell_{I, \bar{s}_{o}}^{=}, R^{j} \Psi_{I, \pi_{o}} \overline{\mathbb{Q}}_{\ell}\right)$, ou plus exactement de leur composante $\Pi^{\infty, o}$-isotypique pour $\Pi$ une représentation automorphe de $D^{\times}(\mathbb{A})$. Boyer montre ensuite que la connaissance de la partie de poids minimal $d-s=s(g-1) \operatorname{des} \mathcal{U}_{\mathcal{L} \mathcal{T}, d}^{d-1-j}\left(S t_{s}\left(\pi_{o}\right)\right)$ suffit à déterminer les termes de la

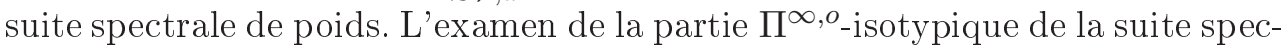
trale des cycles évanescents, pour $\Pi$ telle que $\Pi_{\infty}=S t_{\infty}$ et $\Pi_{o}=S t_{s}\left(\pi_{o}\right)$ puis $\Pi_{o}=\operatorname{Speh}_{s}\left(\pi_{o}\right)$ permet alors de conclure. On utilise pour cela, entre autres, le théorème de Lefschetz difficile et un contrôle obtenu sur la contribution de la strate supersingulière. En fait, on découvre que seule la strate supersingulière contribue en poids $s(g-1)$. On se retrouve donc dans une situation similaire à celle du paragraphe 6.2 .2 où les $E_{2}^{i, j}\left(\Pi^{\infty, o}\right)$ sont nuls sauf pour $i=0$. En définitive, Boyer réussit à exploiter à chaque étape les résultats connus sur la 
cohomologie des faisceaux pervers et les groupes de cohomologie de ces faisceaux, au niveau des diverses suites spectrales en jeu, et à progresser ainsi dans un bon ordre dans des calculs d'apparence inextricables!

La machinerie du côté des corps de nombres opère de la même manière ([16], [15]). Le recours au théorème de monodromie-poids (qui n'est connu qu'en égale caractéristique), s'il a permis de simplifier certains passages de la preuve précédente, peut être contourné, notamment grâce à la dualité de Verdier et à un argument de changement de base dû à Harris. Par contre, on l'obtient en tant que corollaire, pour les variétés de Shimura en question. Signalons également que Boyer présente dans [16] une preuve simplifiée grâce à une information supplémentaire sur la cohomologie de la représentation locale $\mathcal{U}^{i}$ fournie par Fargues ([37]) : en travaillant du côté de la tour de Drinfeld, on peut démontrer un résultat de dualité (issue de la dualité de Poincaré) faisant intervenir l'involution de Zelevinsky entre composantes orthogonales pour l'action de $\mathrm{GL}_{d}(K)$ (indexées par les classes de conjugaison de sous-groupes de Levi, ou encore par le centre de Bernstein [10]). Au contraire, Boyer explique dans [15] comment s'en passer.

\section{Réalisation cohomologique de la correspondance de Langlands pour les représentations elliptiques}

Le théorème 8 montre qu'interviennent dans la cohomologie des deux tours certaines représentations elliptiques de $\mathrm{GL}_{d}(K)$ et que la correspondance de Jacquet-Langlands locale (convenablement étendue, voir remarque cidessous) est réalisée alors que la correspondance de Langlands locale ne l'est que pour les supercuspidales. En travaillant dans la catégorie dérivée des $\overline{\mathbb{Q}}_{\ell}$-représentations lisses de $\mathrm{GL}_{d}(K) \times D_{d}^{\times}$, Dat construit un complexe de cohomologie $R \Gamma_{c}\left(\left(\mathcal{M}^{d}\right)_{\bar{K}}, \overline{\mathbb{Q}}_{\ell}\right)$ qui est plus riche au sens où d'une part il fait intervenir toutes les représentations elliptiques et d'autre part il réalise la correspondance de Langlands (ou plus exactement celle de Hecke, c'est-à-dire la vesion tordue compatible aux automorphismes de $\mathbb{C}$ qui apparait dans la géométrie). 
Théorème 10 (Dat; [23]). - Pour toute représentation lisse irréductible $\pi$ de $\mathrm{GL}_{d}(K)$ on a

$$
\begin{aligned}
H(\pi):= & \bigoplus_{i \in \mathbb{Z}} h^{i}\left(R \operatorname{Rom}_{D^{b}\left(\mathrm{GL}_{d}(K)\right)}\left(R \Gamma_{c}\left(\left(\mathcal{M}^{d}\right)_{\bar{K}}, \overline{\mathbb{Q}}_{\ell}\right), \pi\right)\right) \\
& \simeq \begin{cases}\operatorname{JL}(\pi) \otimes \mathrm{L}(\pi)\left(\frac{d-1}{2}\right) & \text { si } \pi \text { est elliptique } \\
0 & \text { sinon }\end{cases}
\end{aligned}
$$

en tant que représentation de $D_{d}^{\times} \times W_{K}$.

Remarque 11. - 1. On rappelle que les représentations elliptiques (de support la supercuspidale $\pi_{0}$ de $\mathrm{GL}_{d / s}(K)$ et profondeur $s$ ) sont les sous-quotients irréductibles de l'induite $\pi_{0}||^{\frac{1-s}{2}} \times \cdots \times \pi_{0}||^{\frac{s-1}{2}}$. Elles sont paramétrées par les différentes orientations du graphe de sommets $s_{i}=\left(\pi_{0}||^{i-1+\frac{1-s}{2}}\right)_{1 \leq i \leq s}$ et d'arêtes $\left(s_{i}, s_{i+1}\right):$ par exemple, la représentation notée $S_{i, s}\left(\pi_{0}\right)$ dans l'énoncé du théorème 8 correspond à

$$
\stackrel{s_{1}}{\bullet} \rightarrow \stackrel{s_{2}}{\bullet} \rightarrow \ldots \rightarrow \stackrel{s_{i}}{\bullet} \rightarrow \stackrel{s_{i+1}}{\bullet} \leftarrow \ldots \leftarrow \stackrel{s_{s}}{\bullet}
$$

Si $I=\left\{i_{1}, \ldots, i_{k}\right\}$ désigne l'ensemble (ordonné par ordre croissant) des indices des sommets où l'orientation de l'arête $\left(s_{i}, s_{i+1}\right)$ est de gauche à droite, la représentation obtenue, notée $S_{s}^{I}\left(\pi_{0}\right)$, est l'unique sous-module irréductible de l'induite $S t_{n_{0}}\left(\pi_{0}\right)||^{i_{0}+\frac{n_{0}-s}{2}} \times \cdots \times S t_{n_{k}}\left(\pi_{0}\right)||^{i_{k}+\frac{n_{k}-s}{2}}$, où $n_{j}=i_{j+1}-i_{j}\left(0 \leq j \leq k\right.$; on a posé $i_{0}=0$ et $\left.i_{k+1}=s\right)$. En particulier, $S_{i, s}\left(\pi_{0}\right)=S_{s}^{I}\left(\pi_{0}\right)$ avec $I=\{1, \ldots, i\}$. La représentation de $W_{K}$ correspondante est $\mathrm{L}\left(S_{s}^{I}\left(\pi_{0}\right)\right)=S p_{n_{0}}\left(\sigma_{0}\right)\left(i_{0}+\frac{n_{0}-s}{2}\right) \oplus \cdots \oplus S p_{n_{k}}\left(\sigma_{0}\right)\left(i_{k}+\right.$ $\frac{n_{k}-s}{2}$ ) où $\sigma_{0}=\mathrm{L}\left(\pi_{0}\right)$ et la représentation spéciale (généralisée normalisée) $S p_{n}\left(\sigma_{0}\right)$ est $\sigma_{0}\left(\frac{1-n}{2}\right) \oplus \cdots \oplus \sigma_{0}\left(\frac{n-1}{2}\right)$, la monodromie agissant par un bloc de Jordan de taille $n$. Ce sont les sous-modules indécomposables de $\mathrm{L}\left(S_{s}^{I}\left(\pi_{0}\right)\right)$.

2. L'inverse de la correspondance de Jacquet-Langlands induit une injection $R\left(D_{d}^{\times}\right) \hookrightarrow R\left(\mathrm{GL}_{d}(K)\right)$ au niveau des groupes de Grothendieck des représentations de longueur finie. On montre que cette application induit a son tour un isomorphisme $R\left(D_{d}^{\times}\right) \stackrel{\sim}{\rightarrow} \bar{R}\left(\mathrm{GL}_{d}(K)\right)$ sur le quotient $\bar{R}\left(\mathrm{GL}_{d}(K)\right)$ de $R\left(\mathrm{GL}_{d}(K)\right)$ par les combinaisons linéaires d'induites paraboliques. Cela permet de prolonger la correspondance de Jacquet-Langlands en un morphisme JL $: R\left(\mathrm{GL}_{d}(K)\right) \rightarrow R\left(D_{d}^{\times}\right)$et seules les représentations elliptiques ont une image non nulle. Avec les notations précédentes, on a $\left.\left[S_{s}^{I}\left(\pi_{0}\right)\right)\right]=\left[S t_{s}\left(\pi_{0}\right)\right]$ au signe près. 
3. $\mathcal{M}^{d}$ désigne au choix $\mathcal{M}_{\mathcal{L} \mathcal{T}}^{d}$ ou $\mathcal{M}_{\mathcal{D} r}^{d, \dagger}$ qui ont même cohomologie d'après le théorème de Faltings-Fargues (on rappelle que $†$ désigne l'involution $g \mapsto{ }^{t} g^{-1}$ de $\left.\mathrm{GL}_{d}(K)\right)$.

4. Le support de la somme directe est fini : précisément, avec les notations de la première remarque, on a

$\mathcal{H}\left(S_{s}^{I}\left(\pi_{0}\right)\right) \simeq \bigoplus_{j=0}^{k}\left(\mathrm{JL}\left(S t_{s}\left(\pi_{0}\right)\right) \otimes S p_{n_{j}}\left(\mathrm{~L}\left(\pi_{0}\right)\right)\left(i_{j}+\frac{n_{j}-1}{2}+\frac{d-s}{2}\right)\right)[s-k-1+2 j]$

en tant que représentation graduée de $D_{d}^{\times} \times W_{K}$. La beauté du résultat est qu'en définitive les contributions des différents degrés cohomologiques s'assemblent parfaitement!

L'écriture de la suite spectrale de Hochschild-Serre ( $c f$. paragraphe 5.1) a déjà fait intervenir un complexe cohomologique dans la catégorie dérivée des $\mathrm{GL}_{d}(K)$-modules lisses, construit à l'aide d'un recouvrement de Čeck de $\mathcal{M}_{\mathcal{D} r, n}^{d}$ par des ouverts distingués, de nerf l'immeuble de $\mathrm{PGL}_{d}(K)$ ([47] 10.2.2). On a signalé que cette suite spectrale a été généralisée par Fargues, qui s'est inspirée d'idées de Jannsen et Huber afin de traiter des cas où une telle structure simpliciale n'est pas disponible ([35] chap. 4). C'est ce travail qu'adapte Dat pour construire $R \Gamma_{c}$. La construction possède de bonnes propriétés de fonctorialité et peut s'appliquer aussi bien à $\mathcal{M}_{\mathcal{D} r, n}^{d}$ que $\mathcal{M}_{\mathcal{L} \mathcal{T}, n}^{d}$. On passe ensuite à la limite inductive sur $n$.

Dat commence par traiter le cas de la base de la tour, du côté Drinfeld : en d'autres termes, il calcule $\bigoplus_{i \in \mathbb{Z}} h^{i}\left(R H o m_{D^{b}\left(\mathrm{GL}_{d}(K)\right)}\left(R \Gamma_{c}\left(\left(\Omega^{d}\right)_{\bar{K}}, \overline{\mathbb{Q}}_{\ell}\right), \pi\right)\right)$ dans un premier article ([22]). Il s'inspire pour cela du travail de Schneider et Stuhler, dont le formalisme a permis de calculer (entre autres) la cohomologie sans support des $\Omega^{d}$ (la cohomologie avec support ne vérifie par contre pas les axiomes de [65]). Le calcul des $\mathrm{H}_{c}^{i}$ et de leurs extensions mutuelles permet de montrer que le complexe $R \Gamma_{c}$ est scindé : on a $\left.R \Gamma_{c}\left(\left(\Omega^{d}\right)_{\bar{K}}, \overline{\mathbb{Q}}_{\ell}\right) \simeq \bigoplus_{i} \mathrm{H}_{c}^{i}\left(\Omega^{d}\right)_{\bar{K}}, \overline{\mathbb{Q}}_{\ell}\right)[-i]$ dans $D^{b}\left(\mathrm{GL}_{d}(K)\right)$. Un tel scindage induit un isomorphisme entre l'algèbre d'endomorphismes $\operatorname{End}_{D^{b}\left(\mathrm{GL}_{d}(K)\right)} R \Gamma_{c}$ et $\bigoplus_{i \leq j} E x t_{\mathrm{GL}_{d}(K)}^{j-i}\left(\mathrm{H}_{c}^{d-1+j}, \mathrm{H}_{c}^{d-1+i}\right)$ muni du cup-produit, que l'on peut calculer. On obtient une algèbre de matrices triangulaires supérieures et il s'agit d'identifier l'action du groupe de Weil $W_{K}$. D'une part, Dat montre que pour tout relèvement $\widetilde{F r}$ du Frobenius il existe un unique scindage $\widetilde{F r}$-équivariant. D'autre part, il étudie l'action du sous-groupe d'inertie $I_{K}$ à travers l'opérateur $N$ de monodromie (après avoir vérifié que cette action est bien continue). On est ramené à démontrer que $N$ est d'ordre de nilpotence $d$ : pour cela, Dat 
utilise les cycles évanescents associés à un quotient $\widehat{\Omega}^{d} / \Gamma$ par un sous-groupe $\Gamma$ de $\mathrm{PGL}_{d}(K)$ co-compact assez petit (le quotient est alors algébrisable), sur lequel on sait expliciter l'action de $N$.

Pour le cas de la tour entière se posent différents problèmes : afin de suivre la même démarche, il faut d'une part connaître la cohomologie des revêtements. Dat utilise pour cela le résultat de Boyer (théorème 8). D'autre part, le contrôle de la nilpotence de $N$ passe par un modèle formel des revêtements $\Sigma_{n}^{d}$ : or on a déjà fait remarquer qu'on ne sait pas définir dans ce contexte une structure de niveau de Drinfeld. En définitive, c'est du côté de la tour de Lubin-Tate que Dat va œuvrer, le résultat se transférant ensuite par l'isomorphisme de Faltings-Fargues (que Fargues a également rédigé, à la demande de Dat, en terme d'égalités dans la catégorie dérivée).

On considère donc la composante $\rho$-isotypique $R \Gamma_{c}(\rho)$, on montre qu'elle est scindable dans $D^{b}\left(\mathrm{GL}_{d}(K)\right)$ et on suit à peu près le même programme. Pour démontrer que $N$ est d'ordre de nilpotence $s$ lorsque $\rho=\operatorname{JL}\left(S_{s}^{I}\left(\pi_{0}\right)\right)$, Dat est contraint de recourir à une description fine de la géométrie de la variété globale, à travers la fibre $z^{*} \mathrm{R} \Psi \overline{\mathbb{Q}}_{\ell}$ du complexe des cycles évanescents (voir paragraphe 7) en un point supersingulier, fibre qui constitue un $\mathrm{GL}_{d}(K)$-module gradué (avec action de $W_{K}$ ) tel que

$$
z^{*} \mathrm{R} \Psi \overline{\mathbb{Q}}_{\ell} \simeq \bigoplus_{i} \mathrm{H}^{i}\left(\mathcal{M}_{\mathcal{L}}^{d,[0]}, \overline{\mathbb{Q}}_{\ell}\right)[d-1-i]
$$

Il "relève" $z^{*} \mathrm{R} \Psi \overline{\mathbb{Q}}_{\ell}$ en un objet $z_{e q q}^{*} \mathrm{R} \Psi \overline{\mathbb{Q}}_{\ell} \simeq R \Gamma\left(\mathcal{M}_{\mathcal{L} \mathcal{T}}^{d,[0]}, \overline{\mathbb{Q}}_{\ell}\right)[d-1]$ dans $D^{b}\left(\mathrm{GL}_{d}(K)\right)$ et conclut à l'aide de la filtration de monodromie déterminée par Boyer qui fournira le résultat de non annulation. Plus exactement, Dat utilise la bi-filtration (par les noyaux et les images de $N$ ) qui possède la propriété mirifique qu'un bigradué est concentré en un seul degré cohomologique, de sorte qu'il est déteminé dans la catégorie dérivée par sa cohomologie (cela conduira Boyer à utiliser également la bifiltration dans les dernires versions de son article [13]). En définitive, il s'agit d'un travail très élaboré (Dat développe le formalisme catégoriel des faisceaux pervers de Hecke pour construire le foncteur $z_{e ̂ q}^{*}$ et justifier les délicats passages à la limite) qui raffine et complète les résultats de Boyer.

\section{Perspectives}

Michael Harris a énoncé dans ses exposés à ICM 2000 ([43]; voir également [45] §8) et ICM 2002 ([44]) de nombreuses conjectures (certaines sont dues à 
Kottwitz et Rapoport) qui s'apparentent à des généralisations et avatars de la conjecture de Deligne-Drinfeld-Carayol.

Il s'agit d'une part, dans la veine de l'article [46] et en utilisant des techniques rigides-analytiques, de construire des stratifications pour des variétés de Shimura plus générales (de type PEL) et d'étudier les cycles évanescents, les modèle s locaux étant fournis par les espaces de Rapoport-Zink ([60]) qui font intervenir des paires de groupes $(G, J)$, l'un étant une forme intérieure de l'autre. Ce programme a été réalisé en partie par Fargues ([35]) et donne la classification attendue des séries discrètes des deux groupes par le paramêtre de Langlands (paramétrisation encore conjecturale dans le cas général), les deux $L$-paquets étant reliés par une correspondance de type Jacquet-Langlands. Un des problèmes est que l'on ne sait pas définir les généralisations des structures de niveau de Drinfeld nécessaires afin de "déméler" les strates (difficulté qu'à dû contourner Fargues en introduisant une formule des traces de Lefschetz rigide-analytique). Côté corps de fonctions, la théorie des $G$-faisceaux elliptiques ou $G$-chtoucas est encore à élaborer, pour d'autres groupes que $\mathrm{GL}_{d}$, et comprendra son cortège de résultats d'uniformisation, les modèle s locaux étant également à inventer...

D'autre part, on peut considérer la correspondance de Langlands modulo $\ell$ (Vignéras) et réaliser géométriquement ces correspondances (c'était d'ailleurs la motivation initiale de Dat : mieux comprendre cette correspondance pour les séries discrètes). Ou bien encore le cas $\ell=p$ (travaux de Breuil) qui offre un champs d'investigation essentiellement vierge...

Signalons enfin que Faltings a récemment annoncé une généralisation du théorème d'isomorphisme des deux tours à d'autres paires $(G, J)$ que $\left(\mathrm{GL}_{d}, D_{d}^{\times}\right) \ldots$

\section{Références}

[1] Alexandru Ioan Badulescu. Orthogonalité des caractères pour $\mathrm{GL}_{n}$ sur un corps local de caractéristique non nulle. Manuscripta Math., 101(1) :49-70, 2000.

[2] Alexandru Ioan Badulescu. Correspondance de Jacquet-Langlands pour les corps locaux de caractéristique non nulle. Ann. Sci. École Norm. Sup. (4), 35(5) :695-747, 2002.

[3] J. et Deligne-P. Beilinson, A. A. et Bernstein. Faisceaux pervers. In Analysis and topology on singular spaces, I (Luminy, 1981), volume 100 of Astérisque, pages 5171. Soc. Math. France, Paris, 1982.

[4] Vladimir G. Berkovich. Étale cohomology for non-Archimedean analytic spaces. Inst. Hautes Études Sci. Publ. Math., (78) :5-161 (1994), 1993.

[5] Vladimir G. Berkovich. Vanishing cycles for formal schemes. Invent. Math., $115(3): 539-571,1994$. 
[6] Vladimir G. Berkovich. On the comparison theorem for étale cohomology of nonArchimedean analytic spaces. Israel J. Math., 92(1-3) :45-59, 1995.

[7] Vladimir G. Berkovich. Vanishing cycles for formal schemes. II. Invent. Math., 125(2):367-390, 1996.

[8] Vladimir G. Berkovich. p-adic analytic spaces. In Proceedings of the International Congress of Mathematicians, Vol. II (Berlin, 1998), number Extra Vol. II, pages 141-151 (electronic), 1998.

[9] A. V. Bernstein, I. N. et Zelevinsky. Induced representations of reductive $p$-adic groups. I. Ann. Sci. École Norm. Sup. (4), 10(4) :441-472, 1977.

[10] J. N. Bernstein. Le "centre" de Bernstein. In Representations of reductive groups over a local field, Travaux en Cours, pages 1-32. Hermann, Paris, 1984. Edited by P. Deligne.

[11] H. Boutot, J.-F. et Carayol. Uniformisation p-adique des courbes de Shimura : les théorèmes de Čerednik et de Drinfeld. Astérisque, (196-197) :7, 45-158 (1992), 1991. Courbes modulaires et courbes de Shimura (Orsay, 1987/1988).

[12] Jean-François Boutot. Uniformisation $p$-adique des variétés de Shimura. Astérisque, (245) :Exp. No. 831, 5, 307-322, 1997. Séminaire Bourbaki, Vol. 1996/97.

[13] P. Boyer. Faisceaux pervers des cycles évanescents des variétés de Drinfeld et groupes de cohomologie du modèle de Deligne-Carayol. A paraître aux Mémoires de la $S M F$.

[14] P. Boyer. Mauvaise réduction des variétés de Drinfeld et correspondance de Langlands locale. Invent. Math., 138(3) :573-629, 1999.

[15] P. Boyer. Cohomologie des systèmes locaux de Harris-Taylor et applications. Preprint, 2007.

[16] P. Boyer. Monodromie du faisceau pervers des cycles évanescents de quelques variétés de Shimura. Preprint, 2007.

[17] H. Carayol. Nonabelian Lubin-Tate theory. In Automorphic forms, Shimura varieties, and L-functions, Vol. II (Ann Arbor, MI, 1988), volume 11 of Perspect. Math., pages 15-39. Academic Press, Boston, MA, 1990.

[18] Henri Carayol. Sur les représentations $l$-adiques associées aux formes modulaires de Hilbert. Ann. Sci. École Norm. Sup. (4), 19(3) :409-468, 1986.

[19] Henri Carayol. Variétés de Drinfeld compactes, d'après Laumon, Rapoport et Stuhler. Astérisque, (206) :Exp. No. 756, 5, 369-409, 1992. Séminaire Bourbaki, Vol. $1991 / 92$.

[20] Henri Carayol. Preuve de la conjecture de Langlands locale pour $\mathrm{GL}_{n}$ : travaux de Harris-Taylor et Henniart. Astérisque, (266) :Exp. No. 857, 4, 191-243, 2000. Séminaire Bourbaki, Vol. 1998/99.

[21] I. V. Čerednik. Uniformization of algebraic curves by discrete arithmetic subgroups of $\mathrm{PGL}_{2}\left(k_{w}\right)$ with compact quotient spaces. Mat. Sb. (N.S.), 100(142)(1) :59$88,165,1976$.

[22] J.-F. Dat. Espaces symétriques de Drinfeld et correspondance de Langlands locale. Ann. Sci. École Norm. Sup. (4), 39(1):1-74, 2006.

[23] J.-F. Dat. Théorie de Lubin-Tate non-abélienne et représentations elliptiques. Invent. Math., 169(1):75-152, 2007. 
[24] D. et Vignéras M.-F. Deligne, P. et Kazhdan. Représentations des algèbres centrales simples p-adiques. In Representations of reductive groups over a local field, Travaux en Cours, pages 33-117. Hermann, Paris, 1984.

[25] P. Deligne. Les constantes des équations fonctionnelles des fonctions $L$. In $M o$ dular functions of one variable, II (Proc. Internat. Summer School, Univ. Antwerp, Antwerp, 1972), pages 501-597. Lecture Notes in Math., Vol. 349. Springer, Berlin, 1973.

[26] P. Deligne. Lettre à Piatetskii-Shapiro. 1973.

[27] V. G. Drinfeld. Lettre à Carayol. 12 janvier 1980.

[28] V. G. Drinfeld. Elliptic modules. Mat. Sb. (N.S.), 94(136) :594-627, 656, 1974.

[29] V. G. Drinfeld. Coverings of $p$-adic symmetric domains. Funkcional. Anal. i Priložen., 10(2):29-40, 1976.

[30] V. G. Drinfeld. Commutative subrings of certain noncommutative rings. Funkcional. Anal. i Priložen., 11(1) :11-14, 96, 1977.

[31] V. G. Drinfeld. Elliptic modules. II. Mat. Sb. (N.S.), 102(144)(2) :182-194, 325, 1977.

[32] Antoine Ducros. Espaces analytiques p-adiques au sens de Berkovich. Astérisque, (311) :Exp. No. 958, viii, 137-176, 2007. Séminaire Bourbaki. Vol. 2005/2006.

[33] Gerd Faltings. The trace formula and Drinfeld's upper halfplane. Duke Math. J., 76(2) :467-481, 1994.

[34] Gerd Faltings. A relation between two moduli spaces studied by V. G. Drinfeld. In Algebraic number theory and algebraic geometry, volume 300 of Contemp. Math., pages 115-129. Amer. Math. Soc., Providence, RI, 2002.

[35] Laurent Fargues. Cohomologie des espaces de modules de groupes $p$-divisibles et correspondances de Langlands locales. Astérisque, (291) :1-199, 2004. Variétés de Shimura, espaces de Rapoport-Zink et correspondances de Langlands locales.

[36] Laurent Fargues. Application de Hodge-Tate duale d'un groupe de Lubin-Tate, immeuble de Bruhat-Tits du groupe linéaire et filtrations de ramification. Duke Math. J., 140(3) :499-590, 2007.

[37] Laurent Fargues. Dualité de Poincaré et involution de Zelevinsky dans la cohomologie étale équivariante des espaces analytiques rigides. Preprint, 2007.

[38] Laurent Fargues. Filtration de monodromie et cycles évanescents formels. Preprint, 2007.

[39] Laurent Fargues. L'isomorphisme entre les tours de lubin-tate et de drinfeld et applications cohomologiques. In L'isomorphisme entre les tours de Lubin-Tate et de Drinfeld, volume 262 of Progress in Mathematics, pages 3-328. Birkhäuser, 2008.

[40] Alain Genestier. Espaces symétriques de Drinfeld. Astérisque, (234) :ii+124, 1996.

[41] Michael Harris. Supercuspidal representations in the cohomology of Drinfeld upper half spaces; elaboration of Carayol's program. Invent. Math., 129(1) :75-119, 1997.

[42] Michael Harris. The local Langlands conjecture for GL $(n)$ over a $p$-adic field, $n<p$. Invent. Math., 134(1):177-210, 1998. 
[43] Michael Harris. Local Langlands correspondences and vanishing cycles on Shimura varieties. In European Congress of Mathematics, Vol. I (Barcelona, 2000), volume 201 of Progr. Math., pages 407-427. Birkhäuser, Basel, 2001.

[44] Michael Harris. On the local Langlands correspondence. In Proceedings of the International Congress of Mathematicians, Vol. II (Beijing, 2002), pages 583-597, Beijing, 2002. Higher Ed. Press.

[45] Michael Harris. The local Langlands correspondence : notes of (half) a course at the IHP Spring 2000. Astérisque, (298) :17-145, 2005. Automorphic forms. I.

[46] Richard Harris, Michael et Taylor. The geometry and cohomology of some simple Shimura varieties, volume 151 of Annals of Mathematics Studies. Princeton University Press, Princeton, NJ, 2001. With an appendix by Vladimir G. Berkovich.

[47] Thomas Hausberger. Uniformisation des variétés de Laumon-Rapoport-Stuhler et conjecture de Drinfeld-Carayol. Ann. Inst. Fourier (Grenoble), 55(4) :1285-1371, 2005.

[48] Guy Henniart. La conjecture de Langlands locale pour GL(3). In Seminar on Number Theory, Paris 1980-81 (Paris, 1980/1981), volume 22 of Progr. Math., pages 91-105. Birkhäuser Boston, Mass., 1982.

[49] Guy Henniart. Une preuve simple des conjectures de Langlands pour GL $(n)$ sur un corps p-adique. Invent. Math., 139(2) :439-455, 2000.

[50] Guy Henniart. Sur la conjecture de Langlands locale pour $\mathrm{GL}_{n}$. J. Théor. Nombres Bordeaux, 13(1) :167-187, 2001. 21st Journées Arithmétiques (Rome, 2001).

[51] Guy Henniart. Une caractérisation de la correspondance de Langlands locale pour GL(n). Bull. Soc. Math. France, 130(4) :587-602, 2002.

[52] R. P. Jacquet, H. et Langlands. Automorphic forms on GL(2). Lecture Notes in Mathematics, Vol. 114. Springer-Verlag, Berlin, 1970.

[53] Philip Kutzko. The Langlands conjecture for $\mathrm{Gl}_{2}$ of a local field. Bull. Amer. Math. Soc. (N.S.), 2(3) :455-458, 1980.

[54] M. et Stuhler U. Laumon, G. et Rapoport. D-elliptic sheaves and the Langlands correspondence. Invent. Math., 113(2) :217-338, 1993.

[55] John Lubin, Jonathan et Tate. Formal complex multiplication in local fields. Ann. of Math. (2), $81: 380-387,1965$.

[56] John Lubin, Jonathan et Tate. Formal moduli for one-parameter formal Lie groups. Bull. Soc. Math. France, 94 :49-59, 1966.

[57] David Mumford. An analytic construction of degenerating curves over complete local rings. Compositio Math., 24 :129-174, 1972.

[58] G. A. Mustafin. Non-Archimedean uniformization. Uspehi Mat. Nauk, $33(1(199)): 225-226,1978$.

[59] M. Rapoport. On the bad reduction of Shimura varieties. In Automorphic forms, Shimura varieties, and L-functions, Vol. II (Ann Arbor, MI, 1988), volume 11 of Perspect. Math., pages 253-321. Academic Press, Boston, MA, 1990.

[60] Th. Rapoport, M. et Zink. Period spaces for p-divisible groups, volume 141 of Annals of Mathematics Studies. Princeton University Press, Princeton, NJ, 1996.

[61] Michel Raynaud. Construction analytique de courbes en géométrie non archimédienne (d'après David Mumford). In Séminaire Bourbaki, 25e année (1972/1973), 
Exp. No. 427, pages 171-185. Lecture Notes in Math., Vol. 383. Springer, Berlin, 1974.

[62] Michel Raynaud. Géométrie analytique rigide d'après Tate, Kiehl,... In Table Ronde d'Analyse non archimédienne (Paris, 1972), pages 319-327. Bull. Soc. Math. France, Mém. No. 39-40. Soc. Math. France, Paris, 1974.

[63] François Rodier. Représentations de $\mathrm{GL}(n, k)$ où $k$ est un corps $p$-adique. In Bourbaki Seminar, Vol. 1981/1982, volume 92 of Astérisque, pages 201-218. Soc. Math. France, Paris, 1982.

[64] Jonathan D. Rogawski. Representations of $\mathrm{GL}(n)$ and division algebras over a p-adic field. Duke Math. J., 50(1):161-196, 1983.

[65] U. Schneider, P. et Stuhler. The cohomology of $p$-adic symmetric spaces. Invent. Math., 105(1) :47-122, 1991.

[66] Matthias Strauch. On the Jacquet-Langlands correspondence in the cohomology of the Lubin-Tate deformation tower. Astérisque, (298):391-410, 2005. Automorphic forms. I.

[67] John Tate. Rigid analytic spaces. Invent. Math., 12 :257-289, 1971.

[68] Yakov Varshavsky. p-adic uniformization of unitary Shimura varieties. Inst. Hautes Études Sci. Publ. Math., (87) :57-119, 1998.

[69] A. V. Zelevinsky. Induced representations of reductive $p$-adic groups. II. On irreducible representations of GL(n). Ann. Sci. École Norm. Sup. (4), 13(2) :165-210, 1980 .

03 novembre 2008

Thomas Hausberger, I3M - UMR CNRS 5149, cc 051, Université Montpellier 2,

Place Eugène Bataillon, 34095 Montpellier Cedex 5, FRANCE

E-mail : Thomas.hausberger@univ-montp2.fr

Url : http://www.math.univ-montp2.fr/ ${ }^{\text {hausberg }}$ 